\title{
A Cartesian Reflex Assessment of Face Processing
}

\author{
Robert J. Polewan \\ Christopher M. Vigorito \\ Christopher D. Nason \\ University of Massachusetts Amherst \\ Richard A. Block \\ Montana State University \\ John W. Moore \\ University of Massachusetts Amherst
}

Commands to blink were embedded within pictures of faces and simple geometric shapes or forms. The faces and shapes were conditioned stimuli (CSs), and the required responses were conditioned responses, or more properly, Cartesian reflexes (CRs). As in classical conditioning protocols, response times (RTs) were measured from CS onset. RTs provided a measure of the processing cost (PC) of attending to a CS. A PC is the extra time required to respond relative to RTs to unconditioned stimulus (US) commands presented alone. They reflect the interplay between attentional processing of the informational content of a CS and its signaling function with respect to the US command. This resulted in longer RTs to embedded commands. Differences between PCs of faces and geometric shapes represent a starting place for a new mental chronometry based on the traditional idea that differences in $R T$ reflect differences in information processing.

Key Words: eye blink, Cartesian reflex, faces

Familiar things happen, and mankind does not bother about them. It requires a very unusual mind to undertake the analysis of the obvious.

\section{-Alfred North Whitehead, Science and the Modern World (1925)}

In the 1979 film 10, Dudley Moore's character is stopped at a Beverly Hills intersection when he first encounters Bo Derek, her face framed by the window of a limousine en route to her wedding. He is so struck by her beauty that he is slow to react to the change in traffic signals. Faces can compel attention. Some faces are more arresting than others, and it can be difficult to disengage attention and proceed with the workaday task at hand. Imagine the chaos that might ensue if traffic signs were made to resemble faces. "Stop" signs in the United States are simple red octagons for good reason. The framing of the message was selected to enhance the desired effect. Commands for action embedded in a simple geometric shape are naturally complied with more quickly than if the commands were embedded in pictures of faces. This commonsensical observation raises questions about the magnitude or extent of the difference and the nature of contributing factors. We introduce a paradigm, a set of procedures, for addressing a basic question: How long does it take to disengage attention from a picture of a face? There are several possible approaches to this question. The one introduced here involves what we refer to as a Cartesian reflex paradigm (CRP). With it, we report

Authors' Note: We are indebted to Professor Robert S. Feldman of the University of Massachusetts for his encouragement and guidance regarding potential applications of the present research to detecting deception and other domains. We are also indebted to colleague Professor John J. B. Ayres for providing financial support. David S. Torres of Stoughton, Massachusetts, provided invaluable technical advice for the blink data-acquisition system. We thank undergraduate student research assistants Cheryl Berg, Kristy Farinelli, Nicole Duprey, Abby Ingram, Melissa O'Grady, and Stephanie Seiler. Send correspondence concerning this article to John W. Moore, Department of Psychology, University of Massachusetts Amherst, Middlesex House, 111 Country Circle Drive, Amherst, MA 01003; e-mail: jwmoore@psych.umass.edu.

Behavioral and Cognitive Neuroscience Reviews

Volume 5 Number 1, March 2006 3-23

DOI: $10.1177 / 1534582306289130$

(C) 2006 Sage Publications 
that embedding commands in pictures of faces imposes a small but significant cost in reaction time. Because the CRP is closely related to classical conditioning procedures, processes involved in classical conditioning such as anticipation contribute to the magnitude of this effect.

Cartesian reflexes (CRs) are anticipations and false alarms to imperatives to blink or make some otherwise voluntary response in the presence of a signaling or cuing stimulus. The term comes from Descartes' (1911/1967) observation about blinking involuntarily when a friend thrusts his hand toward our eyes in jest. We blink even though we know the friend would not actually strike us. Descartes' example was intended to demonstrate that not all of our behavior is voluntary, the product of human consciousness arising from the soul. Blinking can be a mindless reflex of the brain that channels animal spirits to the muscles that cause the eyelids to close. The act of blinking in Descartes' example bears more than a coincidental resemblance to classical eye-blink conditioning. The conditioned stimulus (CS) is the friend's face at the time of the jest. The hand moving toward the eyes is the unconditioned stimulus (US), and the anticipation of the blow is the conditioned response. ${ }^{1}$ Yet because the blow is never delivered, the reasons for blinking are more complicated than in the case of classical eye-blink conditioning. One cannot but wonder whether previous social interactions between the two friends played a role or whether special circumstances were involved. After all, the blink is a violation of the social contract between these two actors. The friend may be entitled to a harmless jest, but the victim is not necessarily entitled to respond in a manner that betrays a lack of trust. The point of Descartes' example may have been to draw the fine line between voluntary and involuntary action, but its significance also lies in what it tells us about the relationship between the two friends. As psychologists, we would try to ascertain the timing and vigor of the blink. Was it quick and sharp or slow and gradual? We would ask about the appearance of the friend's face. Was it menacing or mirthful? Was the thrusting hand near or far? Did the episode occur as part of an ongoing bout of play or did it occur unexpectedly with no warning?

The CRP is a device for addressing questions about blinking in circumstances similar to Descartes' hypothetical example. A CRP includes a CS such as a face, a blink CR that is voluntary but also subject to involuntary or automatic processes, and a US that elicits a blink but does no actual harm. But the CRP can address more complex issues than those surrounding classical conditioning. Variations in the timing and vigor of the blink may unveil subtle processes such as the distribution of information-processing resources as CS and US vie for control of the participant's attention. The present research demonstrates how the CRP can be exploited to assess information processing, particularly the processing of faces. Information conveyed by another's face often sets the occasion for one's actions and constrains the social transactions between the two parties. More generally, CRPs can directly address questions about how the mind and "animal spirits" interact to control our behavior. Such questions lie at the heart of Descartes' dualistic philosophy. It is why he is widely considered the father of physiological psychology. Although the terminology and methods of modern cognitive neuroscience have advanced, the same questions remain pertinent. The method uses response times (RTs) of commanded actions to make inferences about levels of CS processing. The present research focused on a core problem of early face processing, namely, quantitative assessment of the attentional resources elicited by a face when it is a CS in a CRP.

\section{CRPs and Classical Conditioning}

Traditional eye-blink conditioning procedures that employ air puff USs constitute a subordinate class of CRPs. We extend the definition of CRP to encompass all procedures in which USs are commands to make a voluntary response, with familiar examples being Ivanov-Smolensky's bulb-squeeze experiments (Hartman, 1965) and Hilgard's investigations of eye-blink conditioning based on voluntary blinks as the unconditioned response (UR) (Hilgard \& Marquis, 1940). CRPs meet the operational definition of classical conditioning because participants reliably comply with the US commands. Such compliances are URs. Thus, the term Cartesian reflex can be applied to situations in which the UR is an involuntary response elicited by a "biologically significant" US. It can also be applied to situations in which a voluntary response is elicited by a verbal command or surrogate signal. Surrogate signals are usually employed when participants are instructed to make a voluntary response to some cuing event such as a spoken or written command. The command signal can be preceded by a warning signal that is analogous to an occasion setter or a CS in classical conditioning. The command signals in the CRPs reported here were written English-language prompts appearing on a computer monitor. These signals were designated US commands because of their analogous role as USs.

Because of its superordinate status, the abbreviation $C R$ for Cartesian reflex also encompasses its traditional usage as an abbreviation for conditioned response. Although the letters are the same, the abbreviation $C R$ in this report stands for Cartesian reflex. We contend that CRs can be conditioned responses, even with a voluntary UR, but we do not assert that all CRs are conditioned responses. 
Later on, we propose that responses in the presence of a $\mathrm{CS}$ are to be considered CRs, and responses to US commands presented alone are URs. This convention is one of convenience and clarity of exposition. It need not affect how this research is to be understood or interpreted, although we feel there is a significant connection between processes involved in traditional classical conditioning and the CRP. Indeed, our initial experiments concerned CRs, anticipations, and false alarms arising from CRPs designed to mimic classical conditioning procedures. These experiments are reviewed briefly later on under the heading "Geometric Forms as CSs."

\section{CRPs and Reaction Time}

Procedurally, CRPs resemble classical conditioning, but they also resemble reaction time protocols that involve warning signals (Nickerson, 1973; Welford, 1980). Like classical conditioning and reaction time tasks with warning signals, a CRP is nothing more than a paradigm in which a cue or context sets the occasion for an action triggered by some other stimulus. The main operational distinctions are as follows: (a) Reaction time experiments that employ warning signals often involve variable interstimulus intervals (ISIs) or stimulus onset asynchronies, to use the preferred usage of cognitive psychology (e.g., Logan \& Gordon, 2001). Classical conditioning protocols typically involve a fixed CS-US interval (ISI), although the ISI can differ among various CSs or change occasionally. Unlike reaction time tasks, temporal expectancies and anticipations are not discouraged because they contribute to how a CS is processed. (b) Reaction time experiments often present warning signals without the subsequent command stimulus. These CS-alone trials are catch tests designed to discourage anticipations. They have been shown to induce an inhibitory set or bias that tends to increase response times. Classical conditioning experiments employ CS-alone trials to induce extinction. (c) Reaction time experiments typically employ many trials to attain well-practiced and stable reaction times with a minimum of variability. Classical conditioning experiments typically employ just enough trials so that CRs have time to stabilize and reflect learning. Early trials are not discounted because they reflect orienting responses arising from the initial impact of a CS on the commanded response. Initial reactions can be telling and important in some CRP applications.

Parallels between reaction time and conditioning have long been appreciated (Woodworth \& Schlosberg, 1954 , p. 34). What reaction time and classical conditioning have shared is a reliance on simple stimuli as warning signals and CSs, stimuli such as tones and monochromatic lights of low dimensionality and possessing few distinctive features. The reason for selecting a simple warning cue or CS is that such stimuli are quickly processed, thereby allowing their intended signaling functions to proceed with a minimum of distraction or interference. In the CRP, CSs can be complex, and their capacity to detract from the signaling functions is the point of the exercise. The working hypothesis is that CRs and responses to commands are affected by the information content of the CS and its special relevance to the participant. One consequence is a longer RT to a command. We refer to this additional RT as the processing cost (PC), the cost of attending to and extracting the informational content of a CS instead of merely regarding it as a signal that predicts the command for action.

The commanded action might be facilitated or impeded for many reasons. In CRPs, the ways in which a participant reacts to a command depends not only on imperatives for speed, accuracy, or deliberate caution but also on the makeup of the CS and how its informational content affects the participant. Given the myriad considerations that can reflect subtle and implicit social contracts and cognitions, each individual brings to the paradigm a unique set of histories and tastes about the cuing stimuli in which US commands are set. Psychology provides abundant insights into how human beings might perform to a given set of CSs and USs in the context of a given set of social expectations and relationships. For example, we might expect that participants devote more time attending to a face than to a simple geometric form or shape. Consequently, when faces are used as cuing stimuli, the commanded action would normally be delayed. However, individual participants can deviate from the norm. Instead of being delayed, their action might be facilitated. Such variations in action can be indicative (diagnostic) of the participant's relationship to the person portrayed, the face's emotional impact, or its aesthetic appeal. A CRP can explain a given participant's action only to the extent that it is deemed reasonable in light of particular circumstances and what our science would lead us to expect. From the perspective of traditional principles of classical and instrumental conditioning, we would expect that CRs are dynamic entities that change with experience in a lawful manner. Response timing can be manipulated through instrumental contingencies introduced into a CRP, for example, by rewarding timely and correct responses while withholding rewards when responses are premature, tardy, or inappropriate.

Applying CRPs to the problem of assessing information processing is reminiscent of Wundt's d-reaction experiments circa 1880 (Boring, 1929; Woodworth, 1938). The d-reaction was a reaction time paradigm in which trained observers were required to withhold their response until they had mentally identified and deeply processed the stimulus. A more complex stimulus such 
as a face might result in a longer RT than a simpler stimulus such as a geometric form, and the difference between the two RTs would provide the measure of differential processing time. CRPs also use differential reaction times to infer differences in mental processing but involve no special instructions or training. In time, the d-reaction task was abandoned because observers could not resist reacting immediately to a stimulus, rendering the task a simple RT paradigm.

One advantage of the CRP is that differences in information processing can be assessed without calling on participants to adopt particular sets or attitudes. The CRP experiments described in this report would be considered simple a-reactions, as they do not involve explicit stimulus categorization or response selection processes that characterize the b- and c-reactions of Donders (1868, English-language version reprinted in Koster, 1969; see also Boring, 1929; Teichner \& Krebs, 1974; Woodworth, 1938; Woodworth \& Schlosberg, 1954). The b- and c-reactions arise in choice reaction time paradigms. Although the CRP could be extended to choice reaction time, in the present experiments CRPs were applied to a variation of the simple a-reaction in which a visual warning signal precedes the actiontriggering stimulus. Welford (1980) referred to this paradigm as a sequential reaction-time task. This designation encompasses tasks in which participants are required to react in some way to the first stimulus (S1) before reacting to the second (S2), but the term has also been applied to tasks in which the first stimulus in the sequence does not require an explicit action by the participant (Nickerson, 1967), as was the case here. Implicit information processing of the warning stimulus (S1, CS) can increase the RT to the triggering stimulus (S2, US).

\section{CRPs and Psychological Refractory Periods (PRPs)}

When participants are required to make some response to an S1, and provided the interstimulus interval is on the order of tens or hundreds of milliseconds, a delayed reaction to S2 might be attributed to a PRP or phase, in Telford's (1931) terminology. Smith (1967b) characterized the PRP as follows:

When two stimuli are presented in rapid succession, the reaction time to the second stimulus is typically delayed when compared with the RT to that stimulus when it is presented alone. This delay, the PRP, is maximal when the inter-stimulus interval (ISI) is very short, and declines as the interval between the two stimuli is increased. (p. 125)

In keeping with Smith's characterization, the CRPs in this research employed US-alone trials and varied the
ISI. Smith (1967a) reported that delays in responding to S2 occur when no explicit response to S1 was called for, although the refractory effect was not as great. The largest PRP effects of approximately $200 \mathrm{~ms}$ were observed with an ISI of $50 \mathrm{~ms}$, but they were still evident with ISIs of $600 \mathrm{~ms}$, where they averaged approximately $75 \mathrm{~ms}$. These PRP effects refer to the differences between RTs to S2 when it followed S1 and the RT to S2 when it did not follow S1. Smith (1967a) also compared RTs to S2 when ISI was fixed versus random within blocks of 20 trials. On average, RTs were approximately $200 \mathrm{~ms}$ shorter with fixed ISIs than random ISIs, suggesting a powerful temporal expectancy effect consistent with classical conditioning (see also Adams, 1962). Smith's (1967a) experiment involved a go-no go discrimination to $\mathrm{S} 1$ and a choice discrimination to S2. Furthermore, S1 and S2 were very brief visual stimuli (20 $\mathrm{ms})$, and their location in the visual field signaled which was the required response. Although these complexities did not arise in the present experiments, it is possible that similar PRP effects would materialize.

The CRPs in the present investigation employed ISIs that were within the range of those yielding PRP effects, but they differed in that S2s, the US commands, were embedded within the CSs, the S1s. The CSs were 800 $m s$ in duration, and because the US commands were only $100 \mathrm{~ms}$ in duration, participants could continue CS processing for some time after offset of the US command. The longer study times employed in the CRPs were intended to promote longer latencies in responding to embedded commands than would have been the case with the very brief S1s employed by Smith (1967a, 1967b) and in similar demonstrations of PRP effects. Another factor that would counter PRP effects is anticipations of S2. Such anticipations are CRs. Blinking might terminate CS processing by restricting the view of the CS prematurely. They could even obscure the blink command. Anticipations would be more likely with ISIs conducive to classical conditioning. Such ISIs would be longer than those reported to yield the greatest PRP effects. In sum, although CRPs resemble PRP protocols, there was little a priori concern that PRP effects would significantly undermine our goal of using RT data to infer differences in PCs between faces and geometric shapes as CSs.

\section{Geometric Forms as CSs}

We conducted a series of demonstration experiments with simple geometric forms (shapes) as CSs and commands to blink as USs. The experiments resembled classical conditioning protocols. They were not concerned with information processing. Instead, they explored parameters that affect the probability of anticipations 
(responses that antedate the US command) and false alarms (responses that occur when the US command does not occur). We refer to such responses as Cartesian reflexes (CRs). Whether they are conditioned responses in a Pavlovian sense prompts the question about what constitutes true classical conditioning, particularly human eye-blink conditioning. To fixate on the question of whether CRs are "true" conditioned responses misses the point. The experiments were variations of the basic procedures outlined later in the General Method section. These experiments involved manipulation of stimulus size, durations, salience, and CS-US intervals (ISIs). They also assessed the effects of CS-alone and US-alone trials, as well as the influence of a concurrent distraction task involving tone discrimination. These data guided the protocols employed in the present research. ${ }^{2}$ The main findings were the following: (a) CRs on CS-alone trials were more prevalent with CS-US intervals in the range of 100 to $400 \mathrm{~ms}$ than with shorter or longer ISIs. Furthermore, standard deviations of RTs decreased with ISI, consistent with a shift from control of responding by the CS to control by the blink command. (b) CS-alone trials promoted inhibition of delay, resulting in fewer anticipations and longer response times. (c) The salience of the CS and US (their contrast with surrounds) affected CRs. There were generally more CRs when detection of the US was an issue. (d) RTs were generally longer and more variable to commands embedded in a CS than to those presented alone. (e) Response timing converged quickly over a series of trials with a fixed ISI, thereafter remaining relatively stable.

\section{CRs}

CRPs resemble classical conditioning protocols in that they employ stimuli (CSs and USs) that are presented repeatedly over a series of trials in the manner of Pavlov, typically with a fixed ISI chosen to promote associative learning and the formation of temporal expectations. Because the US commands follow CSs in close temporal proximity, associations and temporal expectations develop quickly. Even though they might not actually precede the appearance of the command, responses faster than URs (responses to US commands presented alone) are CRs because they are under the control of CSs. However, CRs might have longer RTs than URs if they reflect time spent attending to the informational content of a CS as opposed to its predictive value about the occurrence and timing of the US. In either case, we refer to responses in the presence of a CS as CRs. Participants respond differently to US commands embedded in CSs, as attested to by the fact that between-subject variability in RTs is greater on CS trials than on US-alone trials, as described later.
It is difficult to know why a CR might be delayed. One possibility is that processing the content of the CS imposes a switching cost (e.g., Monsell, 2003; Pashler, 1991, 1994; Weichselgartner \& Sperling, 1987). This account assumes that this processing must be completed before attention can shift to the US command. Another possibility is that attention to the CS effectively slows the internal clock that tracks the expected time of the US command (Coull, Vidal, Nazarian, \& Macar, 2004; Zakay \& Block, 1997). By this account, participants learn the ISI and time their responses based on this knowledge, as is typical in eye-blink conditioning (e.g., Sears, Baker, \& Frey, 1979). They learn to react when their internal clock has accumulated sufficient ticks, as it were, not when the command appears on the computer monitor. In other words, their reactions are under the control of the CS, not the US. Attending to the content of the CS instead of its predictive timing function effectively slows the clock and consequently delays the CR.

It might be argued that responses with longer-thannormal reaction times should be considered delayed URs, not CRs. This possibility would accord with the switching-cost account, but for reasons we have just enunciated, it is only when the US command is presented alone that one can be certain that the explicit command and not the CS controls the response. The US command could well be lost by a figurative attentional blink (Olivers \& Nieuwenhuis, 2005) if not a genuine one. Because the mechanisms responsible for delayed responses are not of immediate concern in the present investigation, we refer to all responses occurring in the presence of the CS as CRs no matter what their latency with respect to the US command may be. This convention allows PCs to assume both negative and positive value but does not alter the underlying structure of the task.

\section{Cognitive CS Processing}

What is the cost of processing the information conveyed by the CS? When commands to blink are embedded in pictures of faces, to what extent are RTs sped up or delayed? As outlined above, processing is a term that encompasses attention, but it also includes perception and recognition. In classical conditioning, considerations of CS processing have been limited to a few issues, for example, associability, compound conditioning, discrimination, generalization, and orienting. Here, we focus on the issue of assessing the degree to which a CS consumes attentional resources that would otherwise be devoted to responding to the US in a timely manner. Because the present study concerned face processing, we sought evidence as to how pictures of faces as CSs affected CR timing. We emphasized the blink response 
because, as Descartes intimated, blinking can be influenced by emotional and social factors. In this capacity, eye blinks have diagnostic potential, although not without caveats. Ekman and his associates (e.g., Ekman \& O'Sullivan, 1991; Keltner, Ekman, Gonzaga, \& Beer, 2003) have shown that eye blinks can be useful in detecting deception. Eyelids are controlled by orbicularis oculi muscles. They control microexpressions of emotion that signal deceit or deceptive intent. Exploring connections between eye-blink CRs in protocols involving deceit and deception is a long-term goal.

CRPs provide a novel approach to addressing questions of attentional demands in early stages of information processing-processing that occurs on the order of $100 \mathrm{~ms}$ or less and that is not subject to demands related to detection, recognition, and identification that normally occur within this time frame (GrillSpector \& Kanwisher, 2005). In our usage, the term early processing is not confined to the attention devoted to the CS on its initial presentation. It also refers to attention engaged whenever the CS occurs subsequently over a series of trials. Because levels of attention can change with the passage of time and experience, the number of CS presentations (trials) in a protocol should provide sufficient repetitions for statistical reliability but not so many as to drive CS processing to a minimum because of overexposure. Besides number of presentations, parameters that likely determine the level of attention devoted to a particular CS on any given trial include CS duration and the number of intervening trials from the CS's last presentation (Hintzman, Summers, \& Block, 1975).

\section{CRPs and Information Processing}

Although CRPs can address traditional issues of conditioning and learning using either classical or instrumental procedures, the present research concerned information processing. CRPs can address questions about the costs in intellectual and emotional resources of attending to a CS. The timing of the response reflects this cost. As mentioned previously, $\mathrm{PC}$ is simply the extra time it takes to perform the commanded response when the command is embedded in the CS compared with RTs to commands presented alone. That is, PCs are relative RTs. The historic roots of this approach lie in the single-channel hypothesis of the PRP (see Nickerson, 1967; Welford, 1980). The singlechannel hypothesis assumes that sequential stimuli compete for resources and that this competition affects RTs. A strong version of the single-channel hypothesis, sometimes referred to as a queuing model, assumes that RT can be decomposed into distinct sequential stages, including stimulus and response processing.
Germane to the present investigation, the strong version of the single-channel hypothesis assumes that CS processing precedes instigation of the CR. When a CS elicits a CR with shorter latency than a UR, it does so presumably because information processing is complete and the CS functions solely as a signal. Classical conditioning enhances this signaling function, resulting in CRs that have shorter RTs than they otherwise would. A so-called multichannel hypothesis would allow for information processing of the CS to occur concurrently with response instigation, but restrictions of channel capacity could still apply. CS processing and response instigation can vie for resources, as in an attentional-gate model of timing (Zakay \& Block, 1997). The present investigation was not intended to choose between various instantiations of either a queuing or limited-capacity model, although elements of both might be operating in a particular CRP.

Instead of single-channel constraints, it is conceivable that a delay to the US command occurs because the CS masks the US command, just as the US command or a blink CR could mask or obscure the CS. The command would obscure the CS because of perceptual interference, not because of insufficient processing. To guard against this possibility, US commands in the initial experiments appeared within rectangles that set them off clearly from the CSs. With this precaution, it would have been unlikely that a picture of a face or shape would have masked the blink commands employed as USs. On the other hand, large masks or fonts briefly obscured key features of the CSs such as the eyes in the case of faces.

\section{PCs and Gains}

The single-channel hypothesis assumes that the RT to a CS is the sum of the processing time devoted to the CS, the ISI, and the simple RT to the US command presented alone. Accordingly, the PC for a given participant to a given CS was computed by subtracting the CS-US interval (ISI) and RT to the US alone [RT (US)] from the RT measured from CS onset [RT (CS); see Equation 1]. The RTs in Equation 1 refer to average RTs over trials, not over participants. A positive PC arises whenever RT (CS) exceeds the sum of both the ISI and RT (US). Such a result could well be explained by the PRP imposed by a constrained processing mechanism.

$$
\mathrm{PC}(\mathrm{CS})=\mathrm{RT}(\mathrm{CS})-\mathrm{ISI}-\mathrm{RT}(\mathrm{US}) .
$$

The PC difference between two CSs (CS1 and CS2) for a given participant and ISI is then

$$
\mathrm{PC}(\mathrm{CS} 1)-\mathrm{PC}(\mathrm{CS} 2)=\mathrm{RT}(\mathrm{CS} 1)-\mathrm{RT}(\mathrm{CS} 2) .
$$

Although a positive PC represents a longer than normal RT, a negative PC represents a shorter than normal RT, 
a processing gain. Processing gains occur whenever CRs anticipate the US command. Anticipations may occur because participants overestimate the ISI. They respond prematurely because the metaphorical clock keeping track of elapsed time speeds up.

Negative PCs. Because RTs are measured from CS onsets and not US onsets, it is possible for PCs to be negative. They can indicate that the informational content of a CS is extracted quickly (or ignored) and processing switches to the CS's signaling mode. This rapid switching causes anticipation of the US command. Thus, a processing gain is the time saved in making the target response because of this signaling function. In the experiments reported here, it was not unusual for one CS (typically a face) to express a cost because RTs measured from face onset exceeded RTs to US-alone commands, whereas RTs to another CS (typically a geometric shape) express a gain because RTs measured from shape onset were less than RTs to US-alone commands. The difference between the two RTs is the PC difference, even though one of the CSs expresses a gain, not a loss. Similarly, both CSs could express gains. Nevertheless, the difference in RTs would be a PC difference.

The term processing gain does not refer to the extent or depth of CS processing. It refers only to situations in which CS processing does not delay the response. Indeed, a CS might be processed rapidly enough for participants to anticipate the command and respond quickly. Thorough but rapid CS processing could facilitate anticipation responses if participants attempt to compensate for their internal clock's having been stopped or slowed while the focus of attention was on the content of the CS. A more likely explanation of anticipation responses, however, may be that CS processing is curtailed prematurely because attentional focus is swamped by classical conditioning. In the simplest case, participants would respond quickly to the $\mathrm{CS}$, as if it were a surrogate signal for the US command. This scenario would resemble a strict stimulus substitution view of classical conditioning in which CSs become equivalent to USs in all respects, such that CRs are indistinguishable from URs in latency and other topographical features. Classical eye-blink conditioning, however, typically engages mechanisms of temporal learning, such that CR peak latencies match the timing of USs measured from CS onset. That is, classically conditioned eye-blink CRs typically express temporal specificity (Choi \& Moore, 2003; Kehoe \& Joscelyne, 2005; White, Kehoe, Choi, \& Moore, 2000). Although it is a normal consequence of classical conditioning, this temporal learning can detract from CS processing. Conversely, CS processing may detract from temporal learning or its expression. These ideas are consistent with proposals that motor action involves multiple semi-independent controllers acting in parallel to exert their influence over the motor system (Holroyd \& Coles, 2002).

Positive PCs. In the case of costs, a long delay in responding does not necessarily imply that informational content of a CS receives more thorough processing, although this is a possibility. CSs that impose large PCs may or may not be CSs recognized as familiar later on or have more recognizable features. A large PC does not imply efficient processing, but neither do smaller PCs. Small PCs indicate that participants react quickly. They may respond quickly because they are, in fact, quick to extract the informational content from the CS. Or else they may merely be ignoring the information and using the CS as a preparatory cue for anticipating the command. Similarly, negative PCs, processing gains, do not necessarily imply a lack of processing of CS informational content. They may in fact reflect efficient processing. The question of whether processing costs or gains reflect efficient processing cannot be addressed solely within the CRP framework. Additional evidence might be required. One way to do this is to extend the CRP to include tests of short-term recognition memory. Such tests could compromise or distort the normal range of PCs that would otherwise occur without such demands.

\section{PCs Are Real Numbers}

Unlike simple reaction times, which are always positive numbers, PCs can be positive, zero, or negative. Because they are real-valued ratio-scale measures, PCs can be related directly to other real-valued measures, such as event-related potentials. Furthermore, because RTs are measured from CS onset, not from US onsets (except on US-alone trials), converting RTs effectively extends the range of possible PCs and the PC difference between two CSs. This extension enhances the sensitivity and efficiency of CRPs as tools for assessing the duration of CS processing. Thus, there is a continuum of PCs that extends from negative through zero to positive real numbers. Differences between PCs to faces and shapes (or other frames of references) constitute another perspective on processing times. As noted in Equation 2, the PC difference is simply the difference in RTs measured from CS onsets. If one were not concerned with whether an RT constituted a $\mathrm{PC}$ or a gain, there would be no need for US-alone trials. The extent and direction of the PC difference might suffice. Avoiding US-alone trials could be desirable because unpaired US presentations can impede classical conditioning (Hupka, Kwaterski, \& Moore, 1970; Rescorla, 1968). 


\section{Processing Faces}

Our focus on faces was prompted by an interest in face perception in high-stakes and realistic settings, such as medical diagnostics and security screening. The present investigation did not address questions about the particular aspects of a face that renders it more or less susceptible to scrutiny, although the methodology can be applied to that goal. We employed pictures of faces to illustrate the utility of the CRP for addressing such issues. For example, we explored differences in processing upright and inverted faces, but we might just as well have illustrated the methodology using pictures of upright and inverted automobiles or houses.

In the present experiments, we use the term face processing when a more appropriate characterization might be face-plus or natural face processing. Pictures of faces used in many experiments on face processing conducted by cognitive psychologists are unnatural. They are often monochromatic, subjected to computational and statistical manipulations that emphasize some aspects while obscuring others. They sometimes lack nonfacial features such as hair, eyeglasses, scars and blemishes, neck and shoulders, and attire. In contrast, the natural passport-like pictures of faces we employed were in color. They included hair, neck and shoulders, and aspects of attire. ${ }^{3}$ We chose such natural renderings of faces because a long-term goal is to assess differences among individuals in how they react to pictures of persons they may actually know, not pictures of individuals stripped of key identifying features. Therefore, we chose a more liberal application of the term face processing that encompasses all identifying features normally associated with faces. Furthermore, because pictures of faces were in color, the simple geometric forms we employed as reference CSs were chosen from a spectrum of colors and backgrounds. Faces and forms were selected to promote differences in PCs between stimuli that, although different in complexity, were otherwise visually comparable in size, hues, and saturation.

The present research concerned the difference in costs of processing pictures of faces and simple geometric shapes or forms. The latter have a strong signaling function but little intrinsic informational content. A picture of a face may also possess a strong signaling function while also possessing high informational content, intrinsic appeal, or relevance for the participant. The magnitude of differences in PCs can be telling, and it is important to select CRP parameters that maximize the average difference in PCs between two arbitrary C.Ss. CRPs could be employed to contrast the immediate impact of the Mona Lisa and the Picture of Dorian Grey. However, the magnitude of the difference could be difficult to appreciate without a sense of the effective range of PCs along a continuum extending from the artistically banal to the evocative. Simple geometric forms anchor this continuum. A PC to a simple geometric form provides a baseline for evaluating smaller, subtler differences, such as those between faces expressing different emotional states (Smith, Cottrell, Gosselin, \& Schyns, 2005).

\section{Specific Aims}

The main purpose of the present investigation was to illustrate how CRPs provide a methodology for quantifying information processing in terms of response latency. Processing gains and costs to a CS are expressed in relation to RTs to US commands and also in relation to other CSs. Experiment 1 compared PCs to faces and simple geometric forms using a standard protocol (described below) in which US commands to blink were embedded within faces and forms. The blink command appeared in large letters and on a mask that briefly obscured the CSs. The large font and mask were designed to promote classical conditioning and temporal learning through a US-intensity effect. Experiment 2 explored PCs to faces and forms over a range of CS-US intervals in a withinsubjects design to determine whether CRPs are sensitive to this variable, as are RTs in PRP studies. Experiment 3 resembled Experiment 1, except that blink commands appeared in a small font that did not obscure the CSs. Would similar findings materialize? Experiment 4 compared PCs to upright and inverted faces to determine if CRP protocols are sensitive to this manipulation. Experiment 5 compared PCs to faces and shapes in the context of a short-term recognition memory task.

\section{GENERAL METHOD}

\section{Participants}

One or two consenting participants were run at a time. They were recruited from psychology classes. All protocols were approved by the department's committee for use of human participants, and all experimenters passed university and American Psychological Association requirements for running participants.

Instructions to participants. Participants were told the following, which also appeared on the computer monitor: "Remain seated comfortably and keep looking at the monitor. Do not touch anything during the experiment. You are asked to blink when the word Blink appears. Keep as detached an attitude as possible and simply let your reactions occur naturally. The experiment will begin shortly." At the end of all experiments, participants were requested to "think carefully about the following question before answering. On a scale from 1 to 10 , with 1 being 

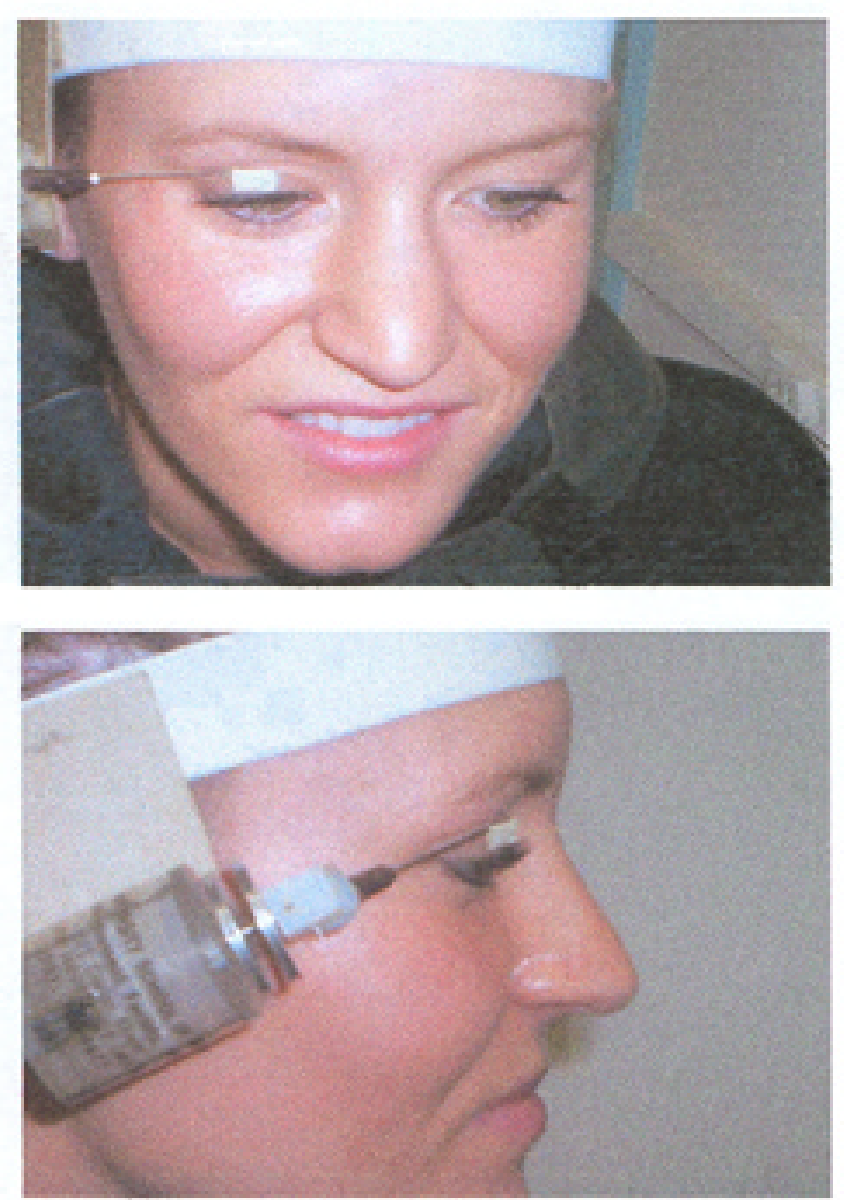

Fgure it Blink Recording Potentiometer and the Method of Attachment to the Participant's Right Upper Eyelid.

NOTE: Tape was affixed to a length of stiff wire and attached to the eyelid. The wire could slide within a tube fashioned from a hypodermic needle to compensate for lateral displacement caused by rotation of the posentiometer.

the lowest and 10 being the highest, how would you' rate your ability to tell what someone is thinking by looking at their face?" A 9-point scale was used in Experiments 4 and 5 , but the two variations vielded the same result. Of 163 participants who rated their face-reading ability, the mean rating was 6.20 , with a standard deviation of 1.39 .

\section{Apparatus and Materials}

Stimuli (CSs and USs) were generated by Cartesio software designed by C. M. Vigorito." Stimuli were presented on Dell Pentium IV computers with 17-in. color monitors, keyboards, and mice, situated on table space sufficient for 2 participants and located in a single $10^{\circ} \times 14^{\prime}$ dimly illuminated room. The experimenter sat nearby to monitor participants' performance and to check to be sure the headgear for recording blinks remained properly situated. Classical music played in the background. As many as 2 participants were run at a time. Experimenters measured the full extensions of participants' right eyelid by hand before and after the experiment to assess whether the head gear became displaced during the experiment. Eye blinks were recorded by a rotary potentiometer affixed to a headband, as illustrated in Figure 1. The potentiometers, designed by Brandon, Logan, Cox, and Wagner (1998), were identical to those used in experiments invoking rabbits (White et al., 2000). Signals from the potentiometer went to an ADC 12 data logger (Pico Technology Limited) plugged into each computer's parallel port and operating with a sampling rate of $250 \mathrm{~Hz}$ (i.e., 4-ms grain).

\section{Protocols}

We embedded commands to blink within pictures of faces and geometric shapes and examined RTs. Our standard protocol involved repeated presentation of two faces and two simple geometric shapes or forms. The same faces and forms were used in Experiments 1 through 4. Each CS was presented for $800 \mathrm{~ms}$ per trial. The command to blink appeared within each CS for $100 \mathrm{~ms}$ and was $100 \mathrm{~ms}$ in duration when presented alone. In Experiments 1 and 2, the font size for the blink command was large, obscuring much of the CS for $100 \mathrm{~ms}$. In Experiments 3 and 4, the font size and framing mask were smaller, revealing key features of the CS. In Experiment 5, the font size was large and presented without a framing mask. In addition, the US command obscured the eyes.

All protocols consisted of 120 trials with varying intertrial intervals of 6,7 , or $8 \mathrm{~s}$, measured from CS offset to next-stimulus onset. Each CS occurred 24 times in a pseudo-random order. Twenty-four US-command. alone trials were interspersed among the 96 CS-US trials. Experiment 2 used a similar protocol except that each of the four CSs was presented with the large-font blink command six times in either an ascending or descending series of CS-US intervals of 50,100, 200, and $400 \mathrm{~ms}$. Unlike other protocols, RTs for US-alone trials were computed locally in blocks of 6 trials. In the other experiments, the ISI for one face and one shape CS was fixed at $200 \mathrm{~ms}$, whereas the ISI was $400 \mathrm{~ms}$ for the other pair of CSs counterbalanced across participants; RTs for US-alone trials were computed globally, that is, across the entire session.

The dependent variable was the peak blink latency obtained by superimposing (point-bypoint summation) the topographical tracings (waveforms) of all trials of a particular type for each individual participant. These peak latencies are typically close to the value of 
the mean obtained by averaging peak latencies from individual trials. The principal effect of employing the peak of superimposed blink topographies is that it gives greater weight to large-amplitude responses. Peak latency RTs are not reaction times, which are typically defined as just-detectable initial excursions (takeoffs) from the recording baseline. It is often difficult to specify initial reaction times within the 4-ms precision of the blink recording system.

\section{Data Presentation}

Because they each involved 30 or more participants, data from Experiments 1, 3, 4, and 5 are presented in tabular form. The tables indicate the mean RTs and PCs (in milliseconds) over participants as functions of stimulus (faces vs. shapes for Experiments 1, 3, and 5 and upright faces vs. the same faces in inverted view for Experiment 4) and ISI (200 vs. $400 \mathrm{~ms}$ ). PC data are also presented graphically. The tables include standard deviations, standard errors, maxima, minima, and medians. Means and standard deviations are included so that readers can readily compute effect sizes and coefficients of variation. Maxima and minima are included to emphasize the ranges of individual variation. Medians are included to indicate the degree of skew in distributions. Generally, medians were within 1 standard error of means, indicating that distributions were not skewed. Tables for Experiments 1, 3, and 5 include mean PC differences between faces (labeled $F$ ) and geometric shapes (labeled $S$ ) for each ISI and the sum of PC differences over ISIs. These entries are labeled DIFF200, DIFF400, and SumDIFF, respectively. When divided by the corresponding standard deviations, these differences are converted to standard scores (effects sizes). In addition, the tables include descriptive statistics of RTs for US-alone trials. Data from Experiment 4 were tabulated the same way but with inverted faces (labeled $I F$ ) replacing shapes. Experiment 2 involved a more complicated procedure in which each CS was presented with each of four ISIs in ascending and descending blocks of trials. RT and PC data from the 8 participants in Experiment 2 are presented graphically.

\section{RESULTS AND DISCUSSION}

\section{Experiment $1(n=34)$ : Blink RTs and PCs for Faces and Shapes With Large-Font US}

Using the protocol described above in the General Method section, Experiment 1 examined blink RTs in the presence of the face and shape CSs shown in Figure 2. The CSs are depicted as they appeared during the 100-ms interval, which included the blink command written in white letters on a black surround. The circle (red) measured 325 pixels in diameter. The square (blue) measured 300 pixels. The faces measured $390 \times$ 444 pixels. The font size for the word Blink was 100 point. The black rectangle measured $260 \times 150$ pixels. Spatially, 100 pixels equaled approximately $30 \mathrm{~mm}$. Thirty-four participants completed this protocol.

PCs and gains. Table 1 and Figure 3 show processing costs and gains. Costs are instances in which RTs to CSs were greater than the sum of the ISI and the RT to blink commands presented alone. Processing gains are negative PCs that arose when RTs to CSs were less than the sum of the ISI and the RT to the blink command presented alone. Table 1 gives mean PCs for each CS and ISI combination across participants. The largest PC was $58 \mathrm{~ms}$, occurring with face CSs and the 200-ms ISI (sign test, $z=4.28, p<.01$, two tailed). Although smaller than typical PRP effects, the PC magnitudes are consistent with RTs for visual recognition reported by GrillSpector and Kanwisher (2005). The other PCs, ranging in value from 19 to $35 \mathrm{~ms}$, were not significantly different from zero by sign tests.

Processing faces versus shapes. Of the 34 participants, 26 had larger PCs to faces than shapes with the 200-ms ISI, and 27 had larger PCs to faces than shapes with the 400-ms ISI. Both differences were statistically significant (sign tests, $z=3.26$ and $z=2.92, p<.01$ ). Because of high variability between participants, however, PC differences between faces and shapes were not significant in terms of parametric tests such as ANOVA. ${ }^{5}$ The mean PC difference between faces and shapes was $31 \mathrm{~ms}$ with the 200-ms ISI $(d=.34)$; the mean PC difference between faces and shapes was $16 \mathrm{~ms}$ with the 400 -ms ISI $(d=.11)$, with effect sizes $(d)$ expressed as $z$ scores in parentheses. Another perspective on differences between PCs of faces and shapes is provided by dividing the mean difference in PCs by the standard deviation of the RT distribution to US alone. This index $\left(d^{\prime}\right)$ regards the RTs to US alone as "noise" in the sense of signal detection theory. With an ISI of $200 \mathrm{~ms}, d^{\prime}=.34$; with the 400-ms ISI, $d^{\prime}=.18$. Strong temporal learning at the expense of CS processing may have contributed to the small effect sizes.

Two factors that may have contributed to strong temporal learning are number of trials and US font size. First, each CS was presented 24 times at a fixed CS-US interval. Second, the large blink command font could have abetted temporal learning to such an extent that it swamped CS processing, a US intensity effect. Furthermore, the large blink command font may have interrupted or truncated face processing prematurely because the key facial features, eyes and nose, were obscured for the 100-ms duration of the blink command. 

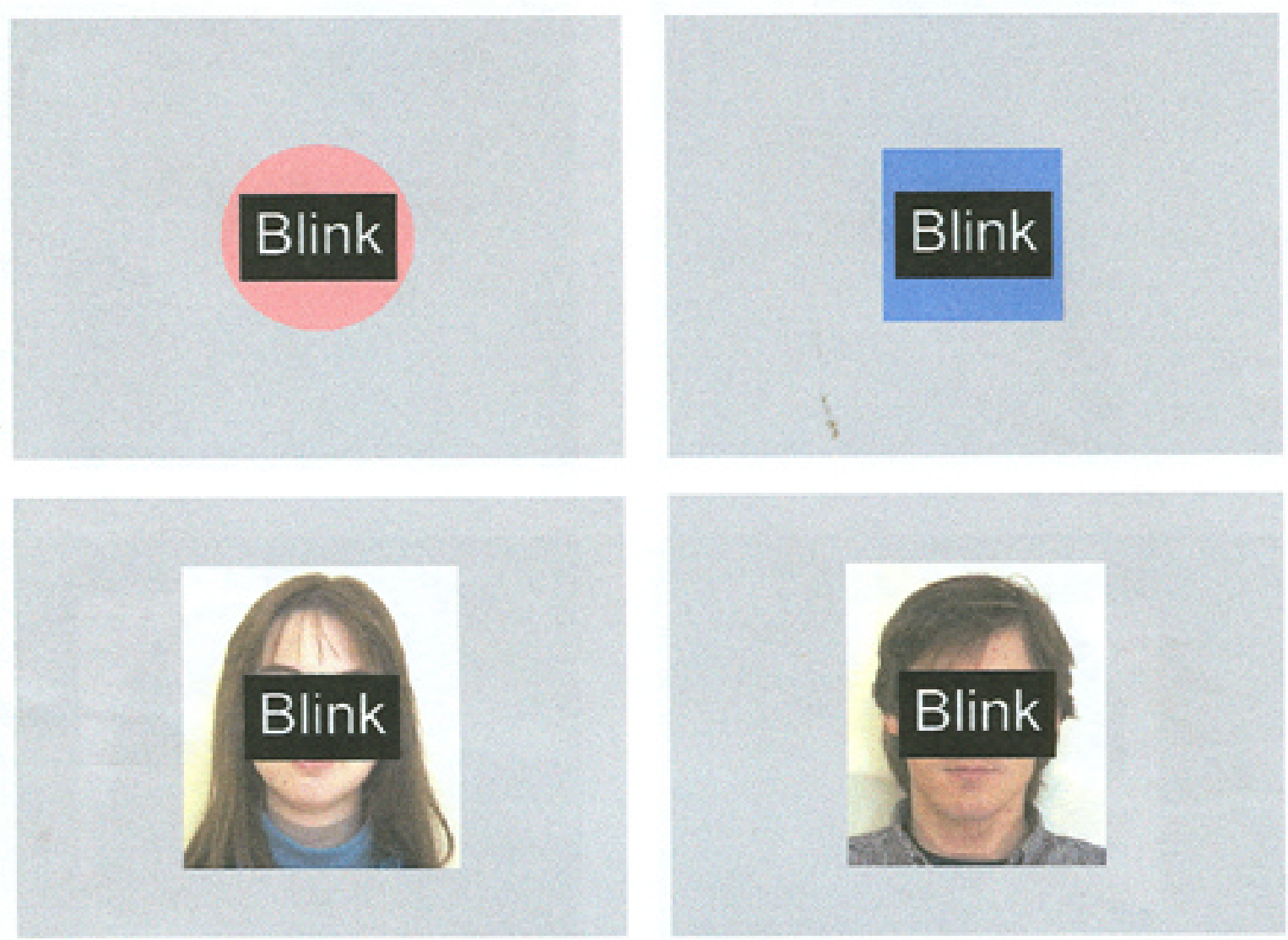

Figure 2s Shape and Face Conditioned Stimuli for Experiment 1 as They Appeared on Compater Moniton at the Time of Unconditioned Stimulua Presentation.

NOTE: The circle was red and the square was blue.

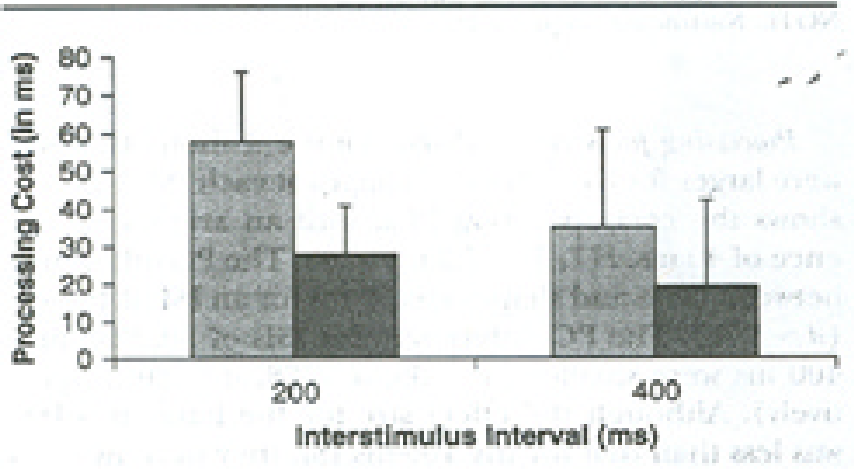

Face $\square$ Shape

Fgure 34 Mean Processing Costs in Milliseconds for Faces and Shapes in Experiment 1 as a Funetion of Interstimmlus Interval. NOTZ: Narrow bars depict 1 SE.
CS control of RTs. Variability in RTs provides an index of the degree to which responses were controlled by CSs instead of US commands. Variability of RTs would be greater in the presence of CSs to the extent that responses occurred before the US commands (anticipations) and afterward because of extended processing delays. The variance $\left(S D^{2}\right)$ of RTs for each CS was contrasted with that of RTs to the US presented alone by forming $F$ ratios (Hays, 1978, pp. 449-451). For faces, RT variability was significantly greater than blink commands alone, for ISIs of 200 and $400 \mathrm{~ms}, F(33,33)=2.72$ and 3.50 , respectively, $p<.01$. For shapes, variability of RTs was significantly greater with the 400 -ms ISI, $F(33,33)=$ $2.65, p<.01$, but not the 200-ms ISI, $F(33,33)=1.68$. $p<10$. In addition, the variability of RTs for faces at the 400 -ms ISI was significantly greater than those for shapes at the 200 -ms ISI, $F(39,33)=2.04, p<.05$. Combined 
Table 1: Blink Response Times and Processing Costs in Milliseconds to Faces and Shapes With Large-Font Blink Command

\begin{tabular}{lccrccc}
\hline Stimulus/ISI & M & SE & SD & Median & Minimum & Maximum \\
\hline Face 200 & 604 & 25 & 148 & 586 & 280 & 892 \\
Shape 200 & 574 & 20 & 116 & 534 & 428 & 868 \\
Face 400 & 781 & 29 & 168 & 772 & 296 & 1045 \\
Shape 400 & 765 & 25 & 146 & 752 & 320 & 1172 \\
US & 346 & 15 & 90 & 340 & 176 & 600 \\
PCF200 & 58 & 18 & 107 & 60 & -316 & 200 \\
PCS200 & 28 & 13 & 75 & 26 & -128 & 204 \\
PCF400 & 35 & 26 & 153 & 48 & -388 & 304 \\
PCS400 & 19 & 23 & 135 & 12 & -476 & 336 \\
Diff200 & 31 & 16 & 92 & 44 & -242 & 280 \\
Diff400 & 16 & 26 & 150 & 60 & -540 & 192 \\
SumDiff & 47 & 37 & 217 & 102 & -484 & 560 \\
\hline
\end{tabular}

NOTE: The top four rows refer to response times (RTs) to conditioned stimuli. The fifth row refers to RTs of unconditioned stimulus (US)-alone trials. Rows 6 to 9 refer to processing costs (PCs) for faces and shapes at the two interstimulus intervals (ISIs). Rows 10 to 12 refer to PC differences between faces and shapes. Entries have been rounded to eliminate decimals. Entries labeled Diff200, Diff400, and SumDiff; PC differences; and the sum of PC differences were rounded after the indicated arithmetic operations.

with the previous comparisons of RTs to blink commands presented alone, one might infer that face CSs exerted more control over the timing of blinks than did shape CSs, and this control was larger with ISIs of 400 than 200 ms. The latter result would be expected if RTs followed Weber's law of timing (White et al., 2000).

\section{Experiment $2(n=8)$ : Blink RTs and PCs for Faces and Shapes With ISIs of $50,100,200$, and $400 \mathrm{~ms}$}

Differences between processing faces and shapes in Experiment 1 were reliable but small. One possible explanation is that the blink command obscured the CSs because of its size (see Figure 2). Furthermore, pairing the large blink command 24 times with each CS at a fixed ISI may have promoted temporal learning to such an extent that it overwhelmed processing differences between faces and shapes. In Experiment 2, we sought to mitigate temporal learning with the large-font blink command by pairing each of the four CSs with the blink command 24 times (as in Experiment 1) but with varying ISIs of 50,100, 200, and $400 \mathrm{~ms}$ in either ascending or descending series. Thus, instead of being held constant as in Experiment 1, ISIs were varied for each CS in an effort to find the most effective ISI for detecting differences between face and shape PCs while at the same time providing sufficient trials for reliable determination of RTs. This stratagem was intended to reduce temporal learning while promoting CS processing.

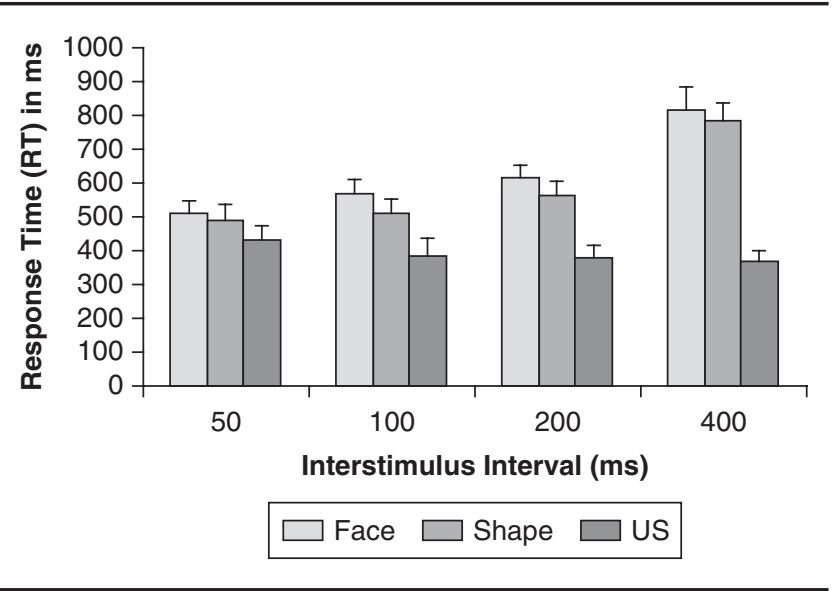

Figure 4: Mean Response Times (RTs) in Milliseconds in Experiment 2 Measured From Conditioned Stimulus (CS) Onsets for Face and Shape CS Together With RTs of Temporally Local Unconditioned Stimulus (US)-Alone Trials, as a Function of Interstimulus Interval.

NOTE: Narrow bars depict 1 SE.

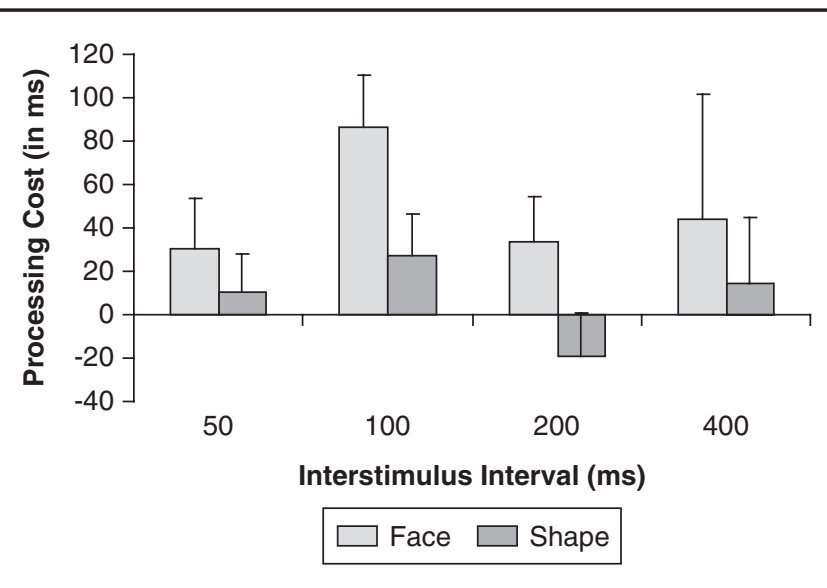

Figure 5: Mean Processing Costs (Gains) in Milliseconds for Face and Shape Conditioned Stimuli as a Function of Interstimulus Interval in Experiment 2.

NOTE: Narrow bars depict 1 SE.

Processing faces versus shapes. Figure 4 shows that RTs were larger for faces than for shapes at each ISI. Figure 5 shows the corresponding PCs, with an average difference of $40 \mathrm{~ms}, F(1,7)=7.33, p=.03$. The PC difference between faces and shapes was $59 \mathrm{~ms}$ for an ISI of $100 \mathrm{~ms}$ $(d=1.78)$. The PC differences for ISIs of 50, 200, and 400 ms were smaller $(d=.38, d=.68, d=.20$, respectively). Although the effect size for the latter two ISIs was less than that for the 100-ms ISI, they were twice as large as the effect sizes for ISIs of 200 and $400 \mathrm{~ms}$ observed in Experiment 1. It would appear that reducing the number of CS-US pairings at a fixed ISI from 24 to 6 effectively reduced temporal learning that may have attenuated PC differences in Experiment 1. 

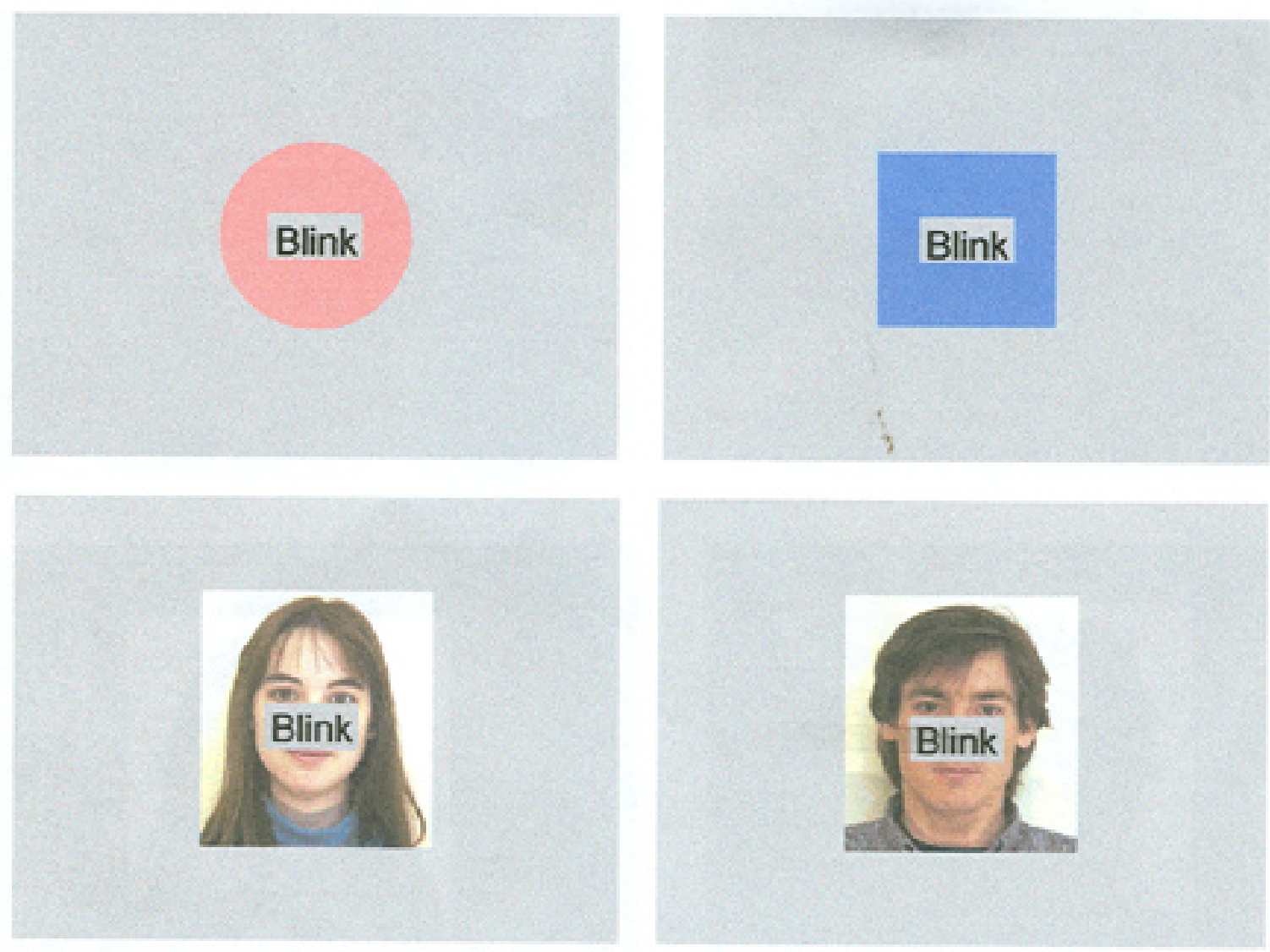

Figure 6: Shape and Face Conditioned Stimuli Used in Experiment 3 Depicted as They Apperared With the 160-ms Blink Command.

\section{Experiment $3(n=36)$ : Blink RTs and PCs for Faces and Shapes With Small-Font US}

Experiment 3 used the same protocol as Experiment 1 but with the small-font blink command illustrated in Figure 6. The CS sizes were the same as in Experiment 1. The blink command appeared in black letters in 65 point framed by a gray rectangle that matched the gray surround of the computer monitor. The gray rectangle measured $169 \times 80$ pixels. The small font was intended to reduce US-intensity effects that may have promoted temporal learning and the expense of processing differences between faces and shapes. As in Experiment 1 , each CS was paired with the US 24 times at a fixed ISI of 200 or 400 ms counterbalanced across participants. Thirty-six participants completed this protocol.

Font size. The smaller font size of the blink command resulted in slower URs on the US-alone trial than observed with the larger fonts employed in Experiments
1 and 2. The mean RT (US) in Experiment 1 was 346 ms $(S E=15)$. In Experiment 3, the mean RT (US) was $406 \mathrm{~ms}(S E=17)$.

PCs and gains. Table 2 shows that RTs to CSs were systematically greater in Experiment 3 than Experiment 1 , and this contributed to larger PCs, particularly for faces (Figure 7). PC differences between faces and shapes were also larger with the small-font US. The mean PCs for faces at the 200 and $400 \mathrm{~ms}$ ISIs, 97 and $62 \mathrm{~ms}$, were significantly greater than zero, sign tests, $z=3.04, p<.01$, and $z=2.36, p<.05$, respectively, and larger than the comparable PCs from Experiments 1 and 2. The PCs for shapes, 51 and $23 \mathrm{~ms}$, were not significantly different from zero. As a main effect from an ANOVA, PCs were greater for faces than shapes, $F(1,35)=13.84, p=.001$. The difference in PCs between ISIs of 200 and $400 \mathrm{~ms}$ was also a significant main effect, $F(1,35)=6.03, p=.019$. The interaction of these two factors was not significant $(F<1)$. 


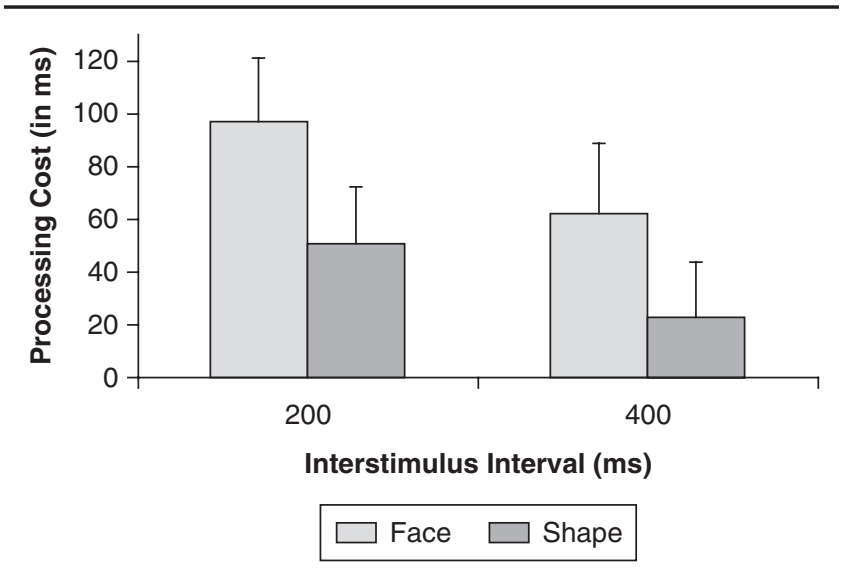

Figure 7: Mean Processing Costs in Milliseconds for Faces and Shapes in Experiment 3 as a Function of Interstimulus Interval.

NOTE: Narrow bars depict $1 S E$.

Consistent with the lack of significant interaction, the mean PC differences between faces and shapes were 47 and $40 \mathrm{~ms}$ for ISIs of 200 and $400 \mathrm{~ms}$, respectively, whereas the corresponding medians were 36 and $48 \mathrm{~ms}$.

\section{Experiment $4(n=31)$ : Blink RTs and PCs for Upright and Inverted Faces}

Experiment 4 assessed PC differences between upright faces and pictures of the same faces in the inverted pose. Figure 8 depicts the face CSs in their upright and inverted orientations. Inverted faces are often used in face perception studies as a means of separating holistic (configural) from componential processes (e.g., Wenger \& Townsend, 2001). From the componential perspective, upright and inverted faces have the same dimensionality. Failure to observe PC differences between upright and inverted faces would indicate that CSs were processed based on their componential complexity and not their holistic or configural aspect.

To the extent that participants have some expertise with both orientations, there should be little difference in PCs between upright and inverted faces (Edelman \& O'Toole, 2001). Upright faces are encountered more often than inverted faces, which are often novel and unexpected. Faces viewed in normal upright orientation impart a greater coalescence of features into a gestalt, with the predicted consequence of smaller PCs. With repeated presentations of the same faces at both orientations, any differences between PCs based on novelty would be attenuated. We had no a priori grounds for predicting that PCs to upright and inverted faces would differ. On one hand, inverted faces are more novel than upright faces are. Novelty could result in additional PCs. On the other hand, inverted faces could be treated as an
Table 2: Blink Response Times and Processing Costs in Milliseconds to Faces and Shapes With Small-Font Blink Command

\begin{tabular}{lrrrrrr}
\hline Stimulus/ISI & M & SE & SD & Median & Minimum & Maximum \\
\hline Face 200 & 704 & 31 & 184 & 700 & 352 & 1072 \\
Shape 200 & 657 & 29 & 176 & 684 & 312 & 972 \\
Face 400 & 869 & 33 & 197 & 908 & 340 & 1264 \\
Shape 400 & 829 & 28 & 170 & 840 & 508 & 1252 \\
US & 406 & 17 & 103 & 378 & 228 & 636 \\
PCF200 & 97 & 24 & 142 & 66 & -140 & 504 \\
PCS200 & 51 & 21 & 126 & 44 & -180 & 408 \\
PCF400 & 62 & 27 & 160 & 70 & -416 & 492 \\
PCS400 & 23 & 21 & 128 & 10 & -192 & 284 \\
Diff200 & 47 & 15 & 92 & 36 & -246 & 344 \\
Diff400 & 40 & 16 & 99 & 48 & -264 & 272 \\
SumDiff & 87 & 23 & 139 & 64 & -232 & 464 \\
\hline
\end{tabular}

NOTE: The top four rows refer to response times (RTs) to conditioned stimuli. The fifth row refers to RTs of unconditioned stimulus (US)-alone trials. Rows 6 to 9 refer to processing costs (PCs) for faces and shapes at the two interstimulus intervals (ISIs). Rows 10 to 12 refer to PC differences between faces and shapes.

unorganized collection of features with little inherent meaning or relevance to participants. As such, their informational content would tend to be ignored, resulting in faster RTs and reduced PCs.

The protocol for Experiment 4 was the same as for Experiment 3, except that inverted faces were employed in place of geometric shapes. Each of the two faces was presented 24 times in each orientation with ISIs of 200 or $400 \mathrm{~ms}$. The ISI was the same for each orientation of a given face. Thirty-one participants completed this protocol.

PCs and gains. Table 3 and Figure 9 show that PCs were greater than zero for both upright and inverted faces. Mean PCs for upright and inverted faces were 70 and $87 \mathrm{~ms}$ with the 200-ms ISI, sign tests, $z=3.60$ and $\mathrm{z}=3.83(p<.01$, two tailed $)$, and 81 and $41 \mathrm{~ms}$ at the 400 ms ISI, sign tests, $z=3.24(p<.01)$ and $2.16(p<.05)$. Although PCs differed significantly from zero, ANOVA failed to reveal PC differences between upright and inverted faces or between ISIs of 200 and $400 \mathrm{~ms}\left(F_{s}<1\right)$. The interaction of these two factors suggests that the two orientations may have been processed differently at the two ISIs, $F(1,30)=2.48, p=.126$. The mean PC difference between upright versus inverted faces was $-17 \mathrm{~ms}$ with the 200-ms ISI and $40 \mathrm{~ms}$ with the $400-\mathrm{ms}$ ISI, suggesting a negligible difference in early processing that was supplanted by greater processing of upright faces than inverted faces with a longer viewing time before the appearance of the blink command. This hypothesis is consistent with effects sizes, which were essentially the same magnitude for the two ISIs but with signs reversed. The effect size of PCs to inverted faces was only slightly 

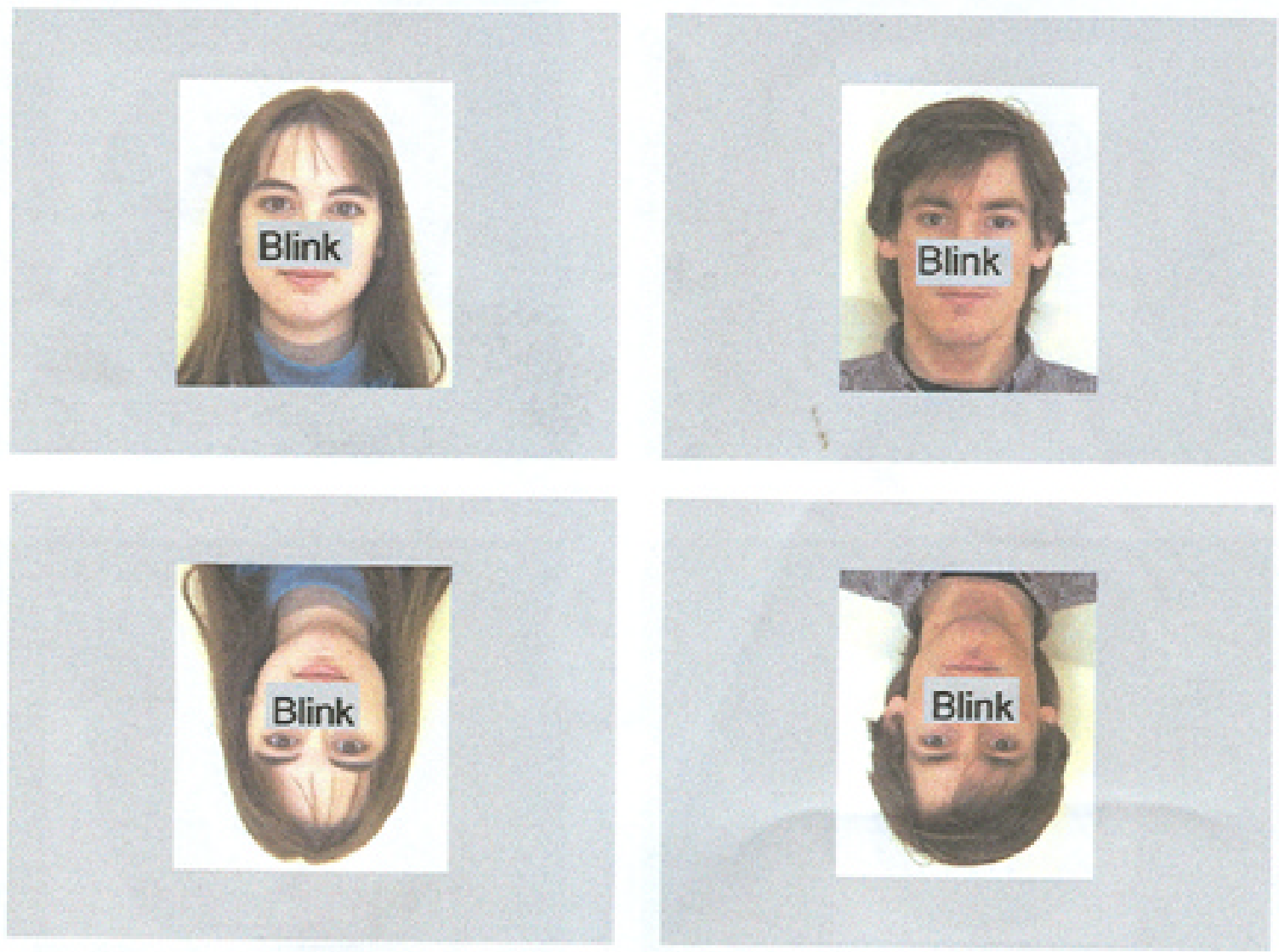

Figure 8: Upright and Inverted Face Conditioned Stimull Used in Experiment 4 Sbowing the Small-Font Blink Commands.

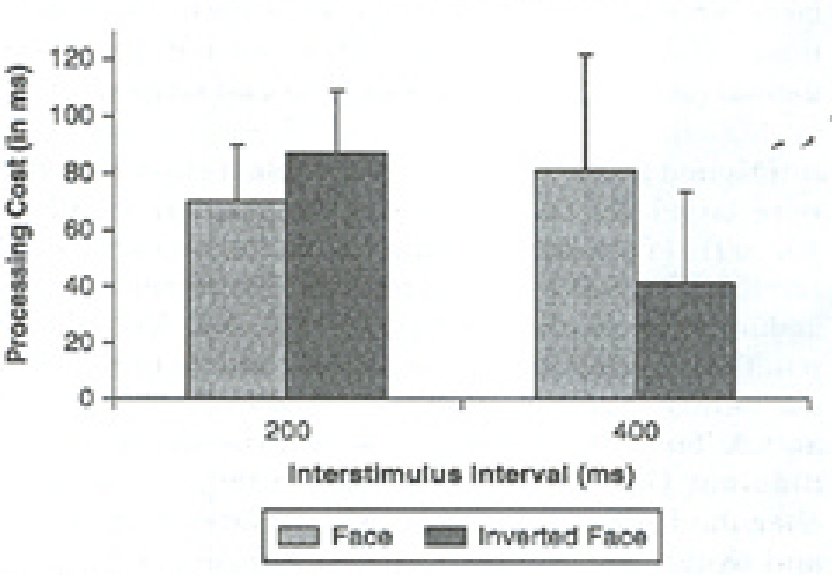

Figure 9: Mean Processing Costs in Maliseconds for Upright and Inverted Faces as a Function of Interstimulus Interval.

NOTE: Narrons bars depict 1 SE. greater than that of PCs to upright faces at an ISI of 200 $\mathrm{ms}(d=-.23)$, but this relationship was reversed at an ISI of $400 \mathrm{~ms}(d=.25)$. (Recall that $d$ is the mean difference in $\mathrm{PC}$ divided by the standard deviation of the difference score.) The effect size with the $400-\mathrm{ms}$ ISI was even larger when expressed as $d^{\prime \prime}$ (mean PC differences divided by the standard deviation of the distribution of RIs to US alone): -.22 and .54 for the $200-$ and $400-\mathrm{ms}$ ISIs, respectively. These observations suggest that differences between upright and inverted faces might be easier to detect with a $400-\mathrm{ms}$ ISI than a 200 -ms ISI. However, the direction of the difference in PCs at this ISI favored inverted faces, suggesting that, like simple geometric shapes, they possess a stronger signaling potential or are not processed as thoroughly as upright faces are.

Table $\mathbf{3}$ shows that RT variability ( $S D$ ) was greater to upright and inverted faces then to blink commands presented alone, with ISIs of both $200 \mathrm{~ms}, F(30,30)=2.42$ 
Table 3: Blink Response Times and Processing Costs in Milliseconds to Upright and Inverted Faces

\begin{tabular}{lrrrrrr}
\hline Stimulus/ISI & M & SE & SD & Median & Minimum & Maximum \\
\hline Face 200 & 648 & 20 & 112 & 636 & 424 & 896 \\
Inverted face 200 & 665 & 24 & 131 & 652 & 392 & 896 \\
Face 400 & 859 & 41 & 227 & 836 & 356 & 1748 \\
Inverted face 400 & 819 & 34 & 188 & 840 & 264 & 1180 \\
US & 378 & 13 & 72 & 364 & 252 & 528 \\
PCF200 & 70 & 20 & 109 & 60 & -192 & 332 \\
PCIF200 & 87 & 22 & 123 & 76 & -192 & 408 \\
PCF400 & 81 & 41 & 231 & 60 & -376 & 1060 \\
PCIF400 & 41 & 33 & 185 & 64 & -468 & 376 \\
Diff 200 & -17 & 13 & 73 & 4 & -244 & 108 \\
Diff 400 & 40 & 29 & 161 & 4 & -300 & 720 \\
SumDiff & 23 & 17 & 150 & 16 & -292 & 644 \\
\hline
\end{tabular}

NOTE: The top four rows refer to response times (RTs) to conditioned stimuli. The fifth row refers to RTs of unconditioned stimulus (US)-alone trials. Rows 6 to 9 refer to processing costs (PCs) for faces and shapes at the two interstimulus intervals (ISIs). Rows 10 to 12 refer to PC differences between upright and inverted faces.

and 3.31, $p<.01$, and $400 \mathrm{~ms}, F(30,30)=9.94$ and 6.82, $p<.01$. However, RT variability for the two face orientations did not differ significantly at either ISI. And although variability of RTs with the 400-ms ISI was generally greater than variability with the $200 \mathrm{~ms}$, as in Experiment 1, the differences were not statistically significant. In short, in terms of RT variability, both face orientations were similar in the control they exerted over responding.

\section{Experiment $5(n=54)$ : Blink RTs and PCs in a Memory Task}

Experiments 1 through 4 employed two faces and two shapes, each presented numerous times to achieve reliable estimates of RTs for individual participants. RTs to blink commands embedded in the faces were longer on average than RTs to blink commands embedded in the shapes. Experiment 5 sought evidence regarding differences in PCs between faces and shapes (see Figure 10) within a memory task. This task involved many CSs. Participants received 120 trials in all, 48 to faces and 48 to shapes, plus 24 blink-alone trials. The number of exposures of a given CS ranged from one to six. On a given trial, the CS was old or new, shown previously or not. After each trial, participants were required to indicate if they had seen the CS previously during the experiment by clicking a computer mouse on boxes labeled old and new.

Half the face trials and half the shape trials had CSUS intervals of $200 \mathrm{~ms}$, and half had CS-US intervals of $400 \mathrm{~ms}$. The CS-US intervals were 200 or $400 \mathrm{~ms}$ but constant for each CS. The RT data reported below come from the second block of 60 trials, at which point the memory load of previously encountered CSs was large and the likelihood of new and old CSs was essentially equal. To achieve reliable estimates, RTs were the averaged peak blink amplitudes over all trials of a particular class: faces versus shapes and old versus new.

Questions of interest included the following: How would the memory task affect RTs to faces and shapes? Would RTs differ for new versus old CSs? How would expanding the stimulus set such that there were fewer trials per CS affect RTs? We had no a priori expectations about these questions. One possible outcome is that the memory task would increase attentional processing of CSs, which could increase PCs. New CSs could receive more processing than old CSs because of novelty. Alternatively, old CSs could receive more processing because of familiarity. With at most six exposures, there would be fewer opportunities for temporal learning, and this could affect PCs.

The experiment was divided into two stages. The first stage consisted of 60 trials: 24 faces, 24 shapes, and 12 blink alone. Sixteen faces and shapes were new (had never been seen before), and 8 faces and shapes had been seen once before. One second after each image disappeared from the screen, two boxes, one containing the word old and the other the word new, appeared. When the participant clicked the mouse in one of these boxes, it briefly turned red, recording the participant's choice. This screen remained on for $3 \mathrm{~s}$ and was followed by a gray screen for a random interval of 2 to $3 \mathrm{~s}$. After this, the next face or shape appeared. In 24 of the 120 trials, the word Blink appeared by itself without a face or form (CS-alone trials).

Stage 2 also contained 60 trials (24 faces, 24 shapes, 12 blink alone). Eight trials had new shapes (had never been seen before). Six trials used shapes that had been presented once before, and 2 contained shapes that had been seen twice. This relationship was also true for faces. One face and one shape were each shown four times. There were four different versions of the experimental protocol with different faces and shapes.

PCs and gains. Figure 11 and Table 4 show that PCs were larger for faces than shapes, $F(1,53)=27.336$, $p<.001$. PCs were also larger with the shorter of the two ISIs, $F(1,53)=44.281, p<.001$. There was an interaction between stimulus type and ISI, $F(1,53)=6.848$, $p=.012$. Apart from the significant interaction, this is the same pattern of results observed in Experiments 1 and 3. Thus, the memory task and the use of several different CSs from both stimulus categories did not alter the basic effect: greater PCs to faces than shapes and to the 200-ms ISI compared with the 400-ms ISI. PC differences between faces and shapes averaged $84 \mathrm{~ms}$ with the $200-\mathrm{ms}$ ISI and $40 \mathrm{~ms}$ with the $400-\mathrm{ms}$ ISI. The effect sizes obtained by dividing each mean difference 

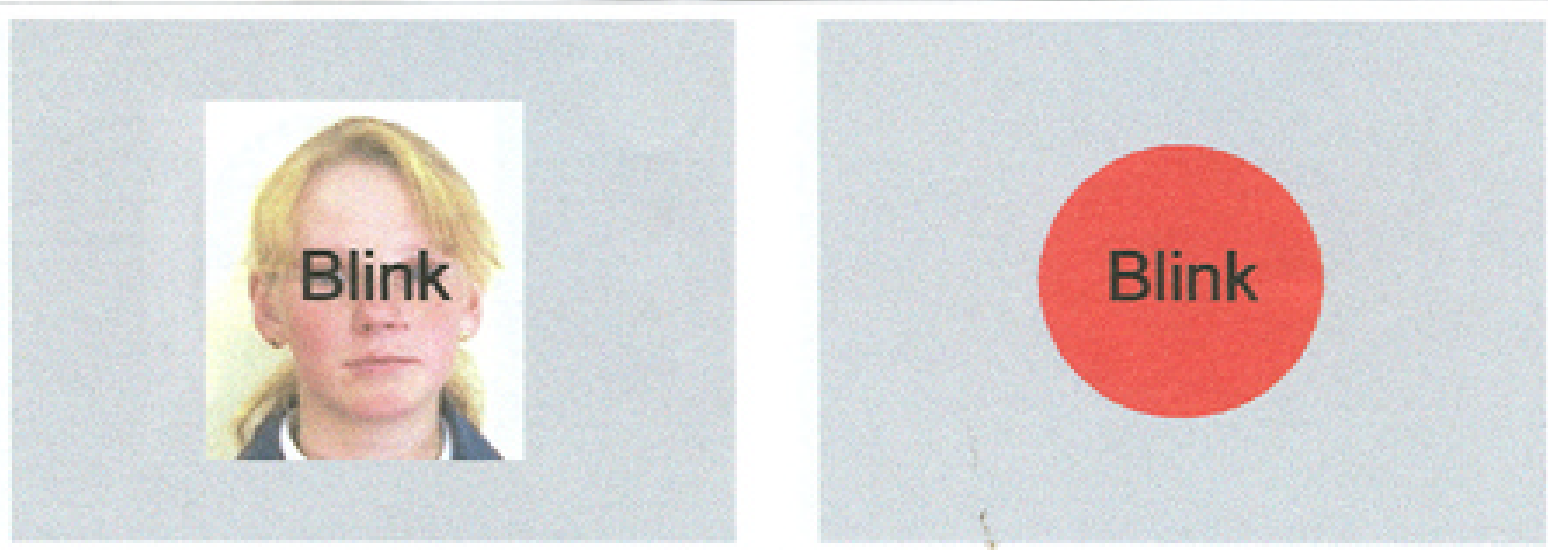

Mgure 10: Shape and Face Conditioned Stimuli Employed in Fxperiment 5 Showing Blink Command.

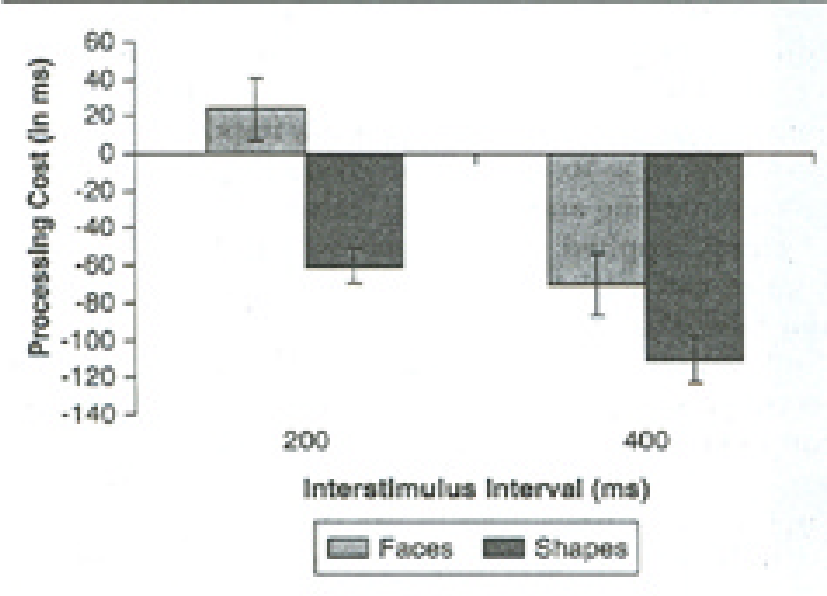

Figure 11: Mean Procesting Costs (Gains) in Milliseconds.

NOTE: Experiment 5 faces and shapes as a function of interstimulus interval, with narrow bars depieting 1 SF:

by the corresponding standard deviation for differences scores were .78 and .38 , which are comparable to those of Experiment 8. This bodes well for applications of CRPs incorporating these features.

Statistical analyses failed to indicate significant differences in processing new and old CSs $(F<1)$. It was not the case that participants could not perform the memory task. The percentage correct classification as new or old for faces during the second half of the experiment was $71 \%$ for faces and $60 \%$ for shapes, a significant difference, $F(1,53)=51.288, p<.001$, which was due to the fact that faces possess more features than shapes. The percentage correct for old-new judgments of faces was significantly correlated with participants' rating of how well they could read faces $(r=.267, p=.05)$. However, the percentage correct for
Thble 4: Blink Response Times and Processing Costs in Milliseconds to Faces and Shapes

\begin{tabular}{|c|c|c|c|c|c|c|}
\hline Stimulnt/TSI & $M$ & SE. & sD & Montien & Míarmurm & Maximurn \\
\hline Face 200 & 680 & 18 & 133 & 648 & 374 & 942 \\
\hline Shape 200 & 566 & 12 & 89 & 562 & 396 & 832 \\
\hline Face 400 & 746 & 17 & 129 & 752 & 478 & 1030 \\
\hline Shape 400 & 716 & 14 & 106 & 712 & 372 & 962 \\
\hline PCres00 & 24 & 17 & 123 & 12 & -506 & $=386$ \\
\hline $\operatorname{PCS} 200$ & -60 & 9 & 66 & -59 & -174 & 120 \\
\hline $\mathrm{PCF} 400$ & -71 & 17 & 122 & -84 & -402 & 274 \\
\hline $\operatorname{PCS400}$ & -111 & 12 & 90 & -112 & -438 & 52 \\
\hline US & 426 & 8 & 62 & 416 & 292 & 528 \\
\hline DHFF200 & 84 & 15 & 108 & 70 & -184 & 442 \\
\hline DIFF 400 & 40 & 14 & 106 & 40 & -284 & 322 \\
\hline SumDiff & 124 & 24 & 174 & 109 & -184 & 764 \\
\hline
\end{tabular}

NOTE: The top four rom refer to reaponse times (RT) to conditioned stimuli. The fifth row refers to RTs of unconditioned stimulus (US)-alone trials. Roms 6 to 9 refer to processing costs (PCs) for faces and shapes at the two interstimulus intervals (ISIs). Rows 10 to 12 refer to PC differences between faces and shapes.

old-new judgments of shapes was not related to selfratings of face-reading ability. Neither memory performance nor ratings of face-reading ability correlated significantly with $\mathrm{PC}$ s or $\mathrm{PC}$ differences.

\section{GENERAL DISCUSSION}

Participants were generally slower to react (longer RTs) when blink commands were embedded within CSs than when presented alone, yielding positive PCs. RTs to shapes were generally faster than RTs to faces. This difference presumably reflects a difference in the attentional demands of extracting the informational content of CSs. These attentional demands are captured by PCs. On average, PCs were langer for faces than for shapes and larger for ISIs of $200 \mathrm{~ms}$ than $400 \mathrm{~ms}$. 
One explanation for the longer RTs to faces than shapes is that processing CSs imposed switching costs (Monsell, 2003) and that these costs were greater for faces than shapes because faces inherently possess more features or information. Processing faces requires more time, and this extra time is the cost of disengaging from a CS's informational content to comply with the commanded action. By this account, attending to the CS would have to be complete before attention can switch to the US command (Pashler, 1991, 1994). The more time devoted to the CS, the longer the RT to the US command. The switching cost account resembles the older single-channel hypothesis of PRPs, discussed previously. An alternative mechanism assumes a trade-off or distribution of attention between processing the content of the CS and attending to the duration of the ISI (Zakay \& Block, 1997). This attentional-gate mechanism implies that participants learn when to expect the US because of experience with a fixed CS-US interval. This information cannot govern accurately the timing of the command if attention is engaged in processing the informational content of the CS. Hence, RTs are longer because the learned temporal estimation of the CS-US interval is compressed. As Coull et al. (2004) expressed it, "Attention modulates our subjective perception of time. The less we attend to an event's duration, the shorter it seems to last" (p. 1506).

One might distinguish between the two interpretations of PCs by considering the effect of ISI on PCs. As with PRP effects, switching costs are typically greater with relatively short ISIs (Pashler, 1991). Information germane to the first task is extracted quickly after a stimulus appears, and the amount of new information diminishes with time. With relatively long ISIs, the attentional load has time to fall to a level that permits unimpeded compliance with US commands. In short, the magnitude of PCs should decrease as the ISI increases. Furthermore, we would expect a greater ISI effect with face CSs than with simple geometric forms, assuming that the latter consume fewer attentional resources than the former do. Differences in PCs between faces and shapes were greater with an ISI of $100 \mathrm{~ms}$ than with ISIs of 50, 200, or $400 \mathrm{~ms}$ in Experiment 2. In Experiment 5, differences were greater at $200 \mathrm{~ms}$ than at $400 \mathrm{~ms}$. The other blink experiments (Experiments 1 and 3) failed to reveal significant PC differences between faces and shapes at ISIs of 200 and $400 \mathrm{~ms}$. Brain imaging using CRPs might distinguish between these alternative interpretations. Recent advances in brain imaging suggest that different neural systems are activated in attentional shifts and temporal estimation (Coull et al., 2004; Shomstein \& Yantis, 2004).

\section{Advantages of Cartesian Reflex Paradigms}

CRPs provide three significant advantages over traditional reaction time approaches to visual information processing.

First, CRs in the present set of experiments were unconstrained. Participants were asked to respond with a blink when the command appeared on the computer monitor. They were not required to detect, categorize, recognize, or identify the CSs. Such requirements could impose additional delays in early stages of visual processing (e.g., Gauthier \& Curby, 2005).

Second, unlike in typical reaction time protocols, participants were not required to respond quickly while avoiding anticipations. Efforts to impose such constraints have proven disappointing, if not futile. Research in the 1960s (e.g., Snodgrass, 1969) aimed at finding "true" RTs by training participants to confine their responses to narrow temporal windows was successful, but these procedures cannot be applied to tasks in which variations in RTs are desirable. Absent constraints to restrict observations so as to conform to theoretically imposed goals, RTs can evolve so as to reflect underlying processes of classical conditioning and temporal learning.

Third, applied to CS processing, CRPs do not require catch trials designed to prevent anticipations and false alarms. Catch trials, presenting a CS without the US, the embedded command, instill an inhibitory set that results in delayed reactions. It is therefore difficult to know whether RTs are long because of this inhibition or because of attentional resources consumed by the CS. Catch trials prevent the very anticipations that are indicative of rapid CS processing. Anticipations are CRs, and they are naturally occurring signals that CS processing may be complete.

\section{Advantages of Simple Geometric Shapes and Forms}

Simple geometric forms and shapes are gestalts. As such, they do not impose the attentional load of complex visual scenes in which dimensionality, segmentation, and expertise contribute to processing times. Circles, squares, and equilateral triangles are gestalts because they recognized and classified quickly. Faces are also gestalts, according to some investigators (Wenger \& Townsend, 2001). Therefore, simple shapes and forms provide an appropriate measuring device for calibrating the costs of processing faces.

We assessed PCs of pictures of real people. CRPs that include less complex materials, such as pseudofaces, would be an exercise in mental chronometry in which contributions of components of faces could be titrated and analyzed against two well-separated points of a 
continuum. As CSs in CRPs, simple geometric forms or shapes possess a clear-cut signaling function with a minimum of superfluous information that can slow RTs. By promoting fast RTs and anticipations, they provide an alternative anchoring point for such an analysis. For example, if the PC for the face were $80 \mathrm{~ms}$, the pseudoface $70 \mathrm{~ms}$, and the shape $30 \mathrm{~ms}$, then the PC for the face can be expressed as either $14 \%$ greater than the pseudoface or $25 \%$ greater, depending on the choice of reference frame. When it comes to assessing cost differentials between faces and pseudofaces, the relative measure could be the more meaningful measure.

Inverted faces. As with the above hypothetical example, PC baselines provided by geometric shapes could be useful in evaluating the magnitude of differences between upright and inverted faces. To illustrate, the mean difference in PCs between upright and inverted faces in Experiment 4 was $40 \mathrm{~ms}$ with an ISI of $400 \mathrm{~ms}$. Upright faces had the larger average PC of $81 \mathrm{~ms}$. This is almost $100 \%$ greater than the average PC for inverted faces of $41 \mathrm{~ms}$. This difference seems even larger when compared with the 23-ms PC for shapes from Experiment 3 (a comparable protocol) at this ISI. Relative to this benchmark, PCs for upright faces were about $200 \%$ greater than for inverted faces. In other words, processing upright faces compared with inverted faces incurred an additional cost of $100 \%$. The additional cost was half the relative differences between upright faces and forms. This example is merely illustrative and does not necessarily foretell the outcome of a CRP that includes shapes as well as upright and inverted faces.

Thus, although it is useful to know the value of a face PC considered in isolation, it can be more useful to specify a PC in terms of a baseline provided by simple geometric forms. The easiest way to do this is to subtract the PC for forms from the PC for faces. This can be done either across experiments with comparable protocols or within experiments whenever participants experience both types of CSs. In the hypothetical example with real and pseudofaces, the PC for forms was subtracted from the PC for faces and pseudofaces. This changed the percentage difference in PCs from $14 \%$ to $25 \%$. A similar subtraction alters one's assessment of the effect of inverting faces. In the above examples, the differences between processing faces and pseudofaces or upright and inverted faces appeared larger when expressed relative to the costs of processing geometric forms or shapes.

ISI effects. PCs are RTs to CSs expressed in relation to RTs to the US command presented alone. As we have seen, PCs can also be expressed in relation to other PCs. As a further illustration, consider the difference between PCs for faces at two ISIs. In Experiment 5, the mean PC for faces was $24 \mathrm{~ms}$ with the 200-ms ISI. The PC for faces with the 400-ms ISI was $-71 \mathrm{~ms}$. The PC difference for faces at the two ISIs was $109 \mathrm{~ms}$. The corresponding PC differences for ISIs with shapes was $51 \mathrm{~ms}$. Relative to shapes, the ISI difference in processing faces was only 44 ms. Employed as a baseline, geometric shapes can alter our perceptions of ISI effects on face processing.

\section{Individual Differences}

In addition to providing a platform for representing PC differences between faces and forms, it is important that CRPs capture individual variations. Indeed, the present experiments emphasize the wide variations in CS processing, with PCs ranging from highly negative to strongly positive. Whether the difference between PCs is large, small, or average may have diagnostic potential or meaning to an informed observer. For example, small differences between PCs for faces and forms may be indicative of efficient face processing. They may be indicative of individuals who read faces quickly and who are capable of detecting fleeting microexpressions of emotion or deceit (Frank \& Ekman, 1997).

As the present experiment demonstrates, differences in PCs between faces and forms can vary considerably among individuals and for different ISIs, thereby providing a temporal signature of PC differences, as illustrated in Figure 12. Factors that determine temporal signatures, ISI-dependent differences in PCs, include the special significance of particular CSs and USs for the participant and the susceptibility of these stimuli to forming associations that promote classical conditioning and temporal learning. Figure 12 shows a typical temporal signature from a participant in Experiment 3. The timing of average (superimposed) blinks with a 200-ms ISI was about $100 \mathrm{~ms}$ greater for faces than shapes, but the two waveforms overlapped with the 400ms ISI. Based on experiments to date, differences in PCs between faces and forms tend to be larger for ISIs in the range of 100 to $200 \mathrm{~ms}$ than for $400 \mathrm{~ms}$, the longest ISI reported here, but individuals differ in this regard. Efficient face processors might evince a significant difference in PCs between faces and forms with short ISIs but not at longer ISIs because, with longer ISIs, US commands may be presented at a time when CS processing is complete or waning. It may be significant that the temporal region of greatest differences in PCs matches the timing of the N170 potential observed in event-related potential studies of early face perception (Gauthier \& Curby, 2005; Schupp et al., 2004).

It remains to be determined whether particular temporal signatures are indicative of efficient face processing. We approached the question of processing efficiency indirectly. Instead of assessing the depth of CS processing directly using a recognition memory task, for 

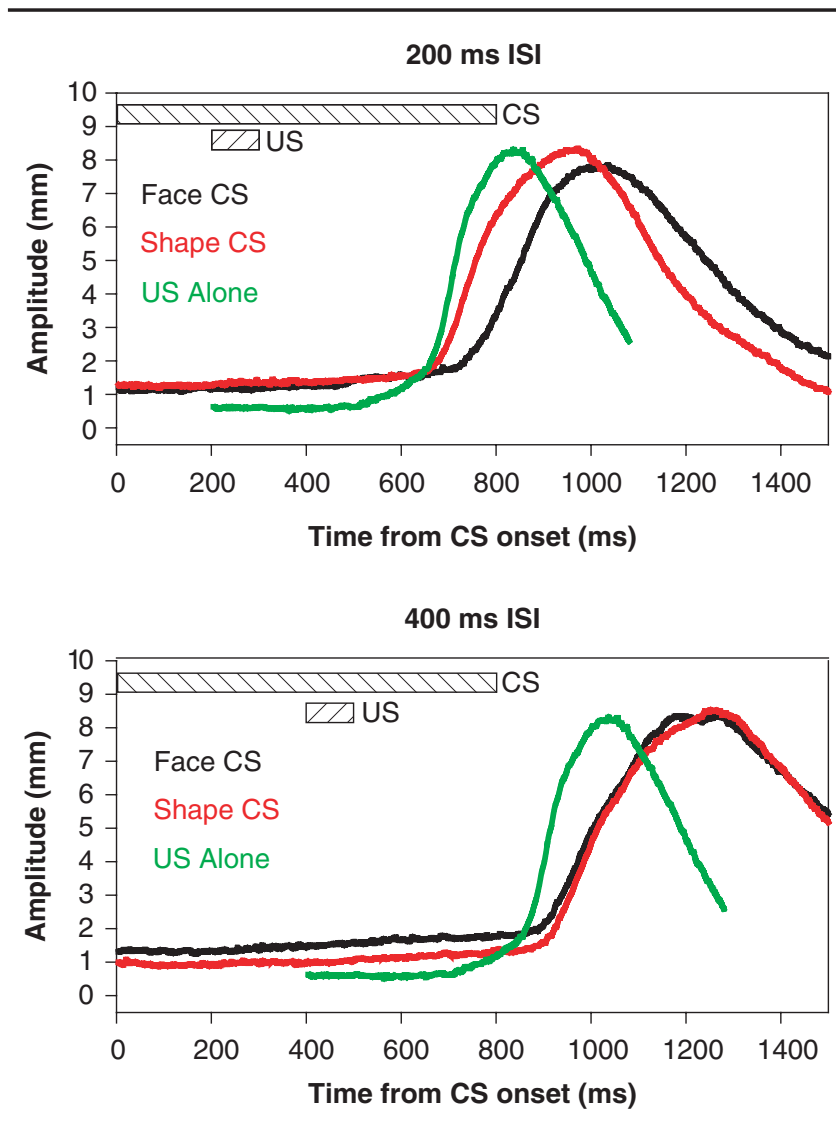

Figure 12: Averaged Blink Waveforms for an Individual Participant From Experiment 3 Showing Responses to Faces, Shapes, and Unconditioned Stimulus (US) Alone at Interstimulus Intervals (ISIs) of 200 ms (Top Panel) and $400 \mathrm{~ms}$ (Bottom Panel).

NOTE: Horizontal bars mark CS and US durations and temporal placement.

example, we solicited participants' assessments of how well they rate their ability to "tell what someone is thinking by looking at their face." This question was asked after each protocol was completed. The idea here is that participants' own assessment of their face-processing ability might relate to the difference between their PCs to faces and to shapes. No statistically significant correlation between self-ratings of face reading and PCs for faces or PC differences between faces and shapes were observed. Self-ratings have proven unreliable guides to accuracy of information extracted from faces. This result is consistent with the literature on self-ratings of ability to detect deceit and deception (Vrij, 2000).

\section{Future Directions}

Factors that govern the timing of CRs extend well beyond the distribution of attentional resources between the content of a cuing stimulus and its role in guiding a timed action, although this is the essence of the matter. Contrasting PCs of faces with simple geometric shapes represents a starting place for a new mental chronometry based on the traditional idea that differences in RT reflect differences in information processing. PCs are ratio-scale variables. So are the differences in PCs between two arbitrary CSs such as face and geometric shape. As such, they can be mapped directly onto other quantitative variables, such as those revealed by modern brain-imaging methods. As with other quantitatively oriented endeavors, it is important to explore the parameters that emphasize these differences and yet produce the greater between-subjects variability. Large baseline differences permit detection of subtle effects. Large intersubject variability is essential for detecting correlations between PCs and the behavioral states encountered in clinical practice, law enforcement, neurology, and psychiatry. Explanations of a particular PC and reasons why it may differ from PCs of other CSs can be inferred from general principles of learning and motivation and from special knowledge of the state and history of the individual.

CRPs are essentially traditional reaction time protocols operating within a broader behavioral framework that encompasses basic processes of associative learning, particularly classical conditioning. The possible application seems boundless, encompassing perception and psychophysics, learning and memory, emotion and motivation, and social cognition. In this regard, CRPs could provide a benign alternative to studies of emotional and social factors in face processing derived from classical conditioning using unpleasant USs such as electric shock (e.g., Öhman \& Dimberg, 1978; Ollson, Ebert, Banaji, \& Phelps, 2005).

\section{NOTES}

1. Those who regard the blink as a purely voluntary action might construe Descartes' example in somewhat different terms. The friend's face would be an occasion setter, the hand moving toward the face would be an immediate threat, and the response would be regarded as an avoidance reaction. Whether the example is cast as classical or instrumental conditioning, or some combination of the two, is not crucial to the present investigation. We adopt the language and perspectives of classical conditioning because Descartes' paradigm resembles classical eye-blink conditioning more than it resembles instrumental avoidance learning.

2. Details are posted on a secured area of the Cartesian reflex paradigm (CRP) Web site labeled Spring 2004 Data $\mathcal{E}$ Reports. Access keys are available on request. The CRP Web address is http://wwwunix.oit.umass.edu/ jwmoore/crp/.

3. Pictures of faces used in this study were obtained from http://pics.psych.stir.ac.uk.

4. Cartesio runs on Windows 98 or Windows XP. Experimenters can select conditioned stimuli from a set of simple geometric shapes or forms (circles, squares, triangles) with a broad selection of size, color, and placement. Cartesio specifies the durations and timing of all events and defined the timing and rate of data logging. Details are available at http://www-unix.oit.umass.edu/ jwmoore/crp/. 
5. The small effect size was due to high between-subjects variability. A repeated-measure ANOVA failed to reject the null hypothesis regarding the reliability of processing cost $(\mathrm{PC})$ differences between faces and shapes. However, the difference was significant by a sign test. Subsequent experiments have consistently demonstrated significant differences in PCs between faces and shapes with ANOVA.

\section{REFERENCES}

Adams, J. A. (1962). Test of the hypothesis of psychological refractory period. Journal of Experimental Psychology, 64, 280-287.

Boring, E. G. (1929). A history of experimental psychology. New York: Century.

Brandon, S. E., Logan, B., Cox, N., \& Wagner, A. R. (1998). A device for concurrent bilateral measurement of the outer eyelid closure in the rabbit. Behavior Research Methods, Instruments, $\mathcal{E}$ Computers, $30,406-410$.

Choi, J.-S., \& Moore, J. W. (2003). Cerebellar neuronal activity expresses complex topography of conditioned eyeblink responses. Behavioral Neuroscience, 117, 1211-1219.

Coull, J. T., Vidal, F., Nazarian, B., \& Macar, F. (2004). Functional anatomy of the attentional modulation of time estimation. Science, 303, 1506-1508.

Descartes, R. (1967). The philosophical works of Descartes (Vol. 1; E. S. Haldane \& G. R. T. Ross, Trans.). Cambridge, UK: Cambridge University Press. (Original work published 1911)

Edelman, S., \& O'Toole, A. J. (2001).Viewpoint generalization in face recognition: The role of category-specific processes. In M. J. Wenger \& J. T. Townsend (Eds.), Computational, geometric, and process perspectives on facial cognition (pp. 397-428). Mahwah, NJ: Lawrence Erlbaum Associates.

Ekman, P., \& O'Sullivan, M. (1991). Who can catch a liar? American Psychologist, 46, 913-920.

Frank, M. G., \& Ekman, P. (1997). The ability to detect deceit generalizes across different types of high-stake lies. Journal of Personality and Social Psychology, 72, 1429-1439.

Gauthier, I., \& Curby, K. M. (2005). A perceptual traffic jam on Highway N170. Current Directions in Psychological Science, 14, 30-33.

Grill-Spector, K., \& Kanwisher, N. (2005). Visual recognition: As soon as you see it, you know what it is. Psychological Science, 16, 152-160.

Hartman, T. F. (1965). Dynamic transmission, elective, generalization, and semantic conditioning. In W. F. Prokasy (Ed.), Classical conditioning (pp. 90-106). New York: Appleton-Century-Crofts.

Hays, W. L. (1973). Statistics for social sciences. New York: Holt, Rinehart and Winston.

Hilgard, E. R., \& Marquis, D. G. (1940). Conditioning and learning. New York: Appleton-Century-Crofts.

Hintzman, D. L., Summers, J. J., \& Block, R. A. (1975). What causes the spacing effect? Some effects of repetition, duration, and spacing on memory for pictures. Memory E Cognition, 3, 287-294.

Holroyd, C. B., \& Coles, M. G. H. (2002). Neural basis of human error processing: Reinforcement learning, dopamine, and the errorrelated negativity. Psychological Review, 109, 679-709.

Hupka, R. B., Kwaterski, S. E., \& Moore, J. W. (1970). Conditioned diminution of the UCR: Differences between the human eyeblink and the rabbit nictitating membrane response. Journal of Experimental Psychology, 83, 45-51.

Kehoe, E. J., \& Joscelyne, A. (2005). Temporally-specific extinction of conditioned responses in the rabbit nictitating membrane response preparation. Behavioral Neuroscience, 119, 1011-1022.

Keltner, D., Ekman, P., Gonzaga, G. C., \& Beer, J. (2003). Facial expression of emotion. In R. J. Davidson, K. R. Scherer, \& H. H. Goldsmith (Eds.), Handbook of affective sciences (pp. 415-432). New York: Oxford University Press.

Koster, W. G. (1969). On the speed of mental processes. Acta Psychologica, 30, 412-431.

Logan, G. D., \& Gordon, R. D. (2001). Executive control of visual attention in dual-task situations. Psychological Review, 108, 393-434.
Monsell, S. (2003). Task switching. Trends in Cognitive Sciences, 7, 134-140.

Nickerson, R. S. (1967). Psychological refractory phase and the functional significance of signals. Journal of Experimental Psychology, 73, 303-312.

Nickerson, R. S. (1973). Intersensory facilitation of reaction time: Energy summation or preparation enhancement? Psychological Review, 80, 489-509.

Öhman, A., \& Dimberg, U. (1978). Facial expressions as conditioned stimuli for electrodermal responses: A case of "preparedness?" Journal of Personality and Social Psychology, 36, 1251-1258.

Olivers, C. N. L., \& Nieuwenhuis, S. (2005). The beneficial effect of concurrent task-irrelevant mental activity on temporal attention. Psychological Science, 16, 265-269.

Ollson, A., Ebert, J., Banaji, M., \& Phelps, E. A. (2005). The role of social groups in the persistence of learned fear. Science, 309, 785-787.

Pashler, H. (1991). Shifting visual attention and selecting motor responses: Distinct attention mechanisms. Journal of Experimental Psychology: Human Perception and Performance, 17, 1023-1040.

Pashler, H. (1994). Dual-task interference in simple tasks: Data and theory. Psychological Bulletin, 116, 230-244.

Rescorla, R. A. (1968). Probability of shock in the presence and absence of CS in fear conditioning. Journal of Comparative and Physiological Psychology, 66, 1-5.

Schupp, H. T., Öhman, A., Junghöfer, M., Weike, A. I., Stockburger, J., \& Hamm, A. O. (2004). The facilitated processing of threatening faces: An ERP analysis. Emotion, 4, 189-200.

Sears, R. J., Baker, J. S., \& Frey, P. W. (1979). The eye blink as a timelocked response. Journal of Experimental Psychology: Animal Behavior Processes, 5, 43-64.

Shomstein, S., \& Yantis, S. (2004). Control of attention shifts between vision and audition in human cortex. Journal of Neuroscience, 24, 10702-10706.

Smith, M. C. (1967a). The psychological refractory period as a function of performance of the first response. Quarterly Journal of Experimental Psychology, 19, 350-352.

Smith, M. C. (1967b). Reaction time to a second stimulus as a function of intensity of the first stimulus. Quarterly Journal of Experimental Psychology, 19, 125-132.

Smith, M. L., Cottrell, G. W., Gosselin, F., \& Schyns, P. G. (2005) Transmitting and decoding facial expressions. Psychological Science, $16,184-189$.

Snodgrass, J. G. (1969). Foreperiod effects in simple reaction time: Anticipation or expectancy? Journal of Experimental Psychology, 79(3 pt. 2), 1-19.

Teichner, W. H., \& Krebs, M. J. (1974). Laws of visual choice reaction time. Psychological Review, 81, 75-98.

Telford, C. W. (1931). Refractory phase of voluntary and associative responses. Journal of Experimental Psychology, 14, 1-35.

Vrij, A. (2000). Detecting lies and deceit. Chichester, UK: John Wiley \& Sons.

Weichselgartner, E., \& Sperling, G. (1987). Dynamics of automatic and controlled visual attention. Science, 238, 778-780.

Welford, A. T. (1980). The single-channel hypothesis. In A. T. Welford (Ed.), Reaction times (pp. 215-252). New York: Academic Press.

Wenger, M. J., \& Townsend, J. T. (2001). Faces as Gestalt stimuli: Process characteristics. In M. J. Wenger \& J. T. Townsend (Eds.), Computational, geometric, and process perspectives on facial cognition (pp. 229-284). Mahwah, NJ: Lawrence Erlbaum Associates.

White, N. E., Kehoe, E. J., Choi, J.-S., \& Moore, J. W. (2000). Coefficients of variation in timing of the classically conditioned eyeblink in rabbits. Psychobiology, 28, 520-524.

Woodworth, R. S. (1938). Experimental psychology. New York: Holt.

Woodworth, R. S., \& Schlosberg, H. (1954). Experimental psychology. New York: Holt.

Zakay, D., \& Block, R. A. (1997). Temporal cognition. Current Directions in Psychological Science, 6, 12-16. 